Pacific

Journal of

Mathematics

CAUSAL COMPACTIFICATION AND HARDY SPACES FOR SPACES OF HERMITIAN TYPE

Frank Betten and Gestur Ólafsson 


\title{
CAUSAL COMPACTIFICATION AND HARDY SPACES FOR SPACES OF HERMITIAN TYPE
}

\author{
Frank Betten and Gestur Ólafsson
}

Let $G / H$ be a compactly causal symmetric space with causal compactification $\Phi: G / H \rightarrow \check{S}_{1}$, where $\check{S}_{1}$ is the BergmanSilov boundary of a tube type domain $G_{1} / K_{1}$. The Hardy space $\mathrm{H}_{2}(C)$ of $G / H$ is the space of holomorphic functions on a domain $\Xi\left(C^{o}\right) \subset G_{\mathbb{C}} / H_{\mathbb{C}}$ with $L^{2}$-boundary values on $G / H$. We extend $\Phi$ to imbed $\Xi\left(C^{o}\right)$ into $G_{1} / K_{1}$, such that $\Xi\left(C^{o}\right)=$ $\left\{z \in G_{1} / K_{1} \mid \psi_{m}(z) \neq 0\right\}$, with $\psi_{m}$ explicitly known. We use this to construct an isometry $I$ of the classical Hardy space $H_{c l}$ on $G_{1} / K_{1}$ into $H_{2}(C)$ or into a Hardy space $\widetilde{H}_{2}(C)$ defined on a covering $\widetilde{\Xi}\left(C^{o}\right)$ of $\Xi\left(C^{o}\right)$. We describe the image of $I$ in terms of the highest weight modulus occuring in the decomposition of the Hardy space.

\section{Introduction.}

Hardy spaces on tube type domains $T_{\Omega}=\mathbb{R}^{n}+i \Omega$, associated to a homogeneous self dual cone $\Omega \subset \mathbb{R}^{n}$, are important objects in analysis. The Hardy space $H_{c l}$ is by definition the space of holomorphic functions on $T_{\Omega}$, such that the Hardy norm

$$
\|f\|_{2}^{2}:=\sup _{y \in \Omega} \int_{\mathbb{R}^{n}}|f(x+i y)|^{2} d x
$$

is finite $([\mathbf{S W 7 1}, \mathbf{F K 9 4}])$. The boundary value map $\beta: H_{c l} \rightarrow L^{2}\left(\mathbb{R}^{n}\right)$ is given by

$$
\beta(f)(x)=\lim _{y \rightarrow 0} f(x+i y),
$$

the limit taken in the $L^{2}\left(\mathbb{R}^{n}\right)$-norm. The image of $\beta$ is described by the positivity condition

$$
\operatorname{Im}(\beta)=\mathcal{F}\left(L^{2}(\Omega)\right),
$$

where $\mathcal{F}$ is the Fourier transform and $L^{2}(\Omega)$ the space of $L^{2}$-functions supported on $\Omega \subset \mathbb{R}^{n}$. The evaluation map $H_{c l} \ni f \mapsto f(w) \in \mathbb{C}, w \in T_{\Omega}$, is continuous, and thus given by an element $K_{w} \in H_{c l}$. The function $K(z, w):=K_{w}(z)$ is the Cauchy kernel associated to the tube domain $T_{\Omega}$. The Cauchy kernel is determined by a function of one variable $K(z, w)=$ 
$K(z-\bar{w})$, where $K(z)$ is the Laplace transform of the characteristic function of the cone. Finally the inverse of the boundary value map is

$$
f(z)=\int_{\mathbb{R}^{n}} \beta(f)(y) K(z-y) d y .
$$

For us the tube domain $T_{\Omega}$ is allways the unbounded realization of a Hermitian symmetric space $G_{1} / K_{1}$ such that $H_{c l}$ is also a $G_{1}$-representation space $([\mathbf{F K 9 4}])$.

The notation of Hardy spaces was generalized to compactly causal symmetric spaces $G / H$ ([HÓØ91]). In this case a complex manifold $\Xi\left(C^{o}\right) \subset$ $G_{\mathbb{C}} / H_{\mathbb{C}}$, depending on the causal structure $m \mapsto C(m)$ on $G / H$, was constructed. Three important properties of $\Xi\left(C^{o}\right)$ are:

(1) The manifold $\Xi\left(C^{o}\right)$ is locally isomorphic to a tube domain $\mathfrak{q}+i C^{o}$, where $\mathfrak{q}$ is the tangent space of $G / H$ at $x_{o}=e H$ and $C^{o}$ the interior of $C:=C\left(x_{o}\right)$.

(2) The homogeneous space $G / H$ is a boundary component of $\Xi\left(C^{o}\right)$.

(3) There is a semigroup $\Gamma$ containing $G$ and determined by $C$ such that $\Xi\left(C^{o}\right)=\left(\Gamma^{o}\right)^{-1} \cdot x_{o}$.

The Hardy space $H_{2}(C)$ is defined as in the classical case to be the space of holomorphic functions on $\Xi\left(C^{o}\right)$ such that the Hardy norm

$$
\|f\|_{H}^{2}:=\sup _{\gamma \in \Gamma^{o}} \int_{G / H}\left|f\left(\gamma^{-1} \cdot m\right)\right|^{2} d m
$$

is finite. $H_{2}(C)$ is a Hilbert space with norm $\|\cdot\|_{H}$, and - as in the classical case - there exists an isometry $\beta: H_{2}(C) \rightarrow L^{2}(G / H)$ given by

$$
\beta(f)=\lim _{\Gamma^{\circ} \ni \gamma \rightarrow 1} \gamma \cdot f,
$$

where the limit is in $L^{2}(G / H)$ and $\gamma \cdot f(\xi):=f\left(\gamma^{-1} \cdot \xi\right)$. The left action defines a holomorphic representation $T$ of $\Gamma$ on the Hardy space and a unitary representation $\lambda$ of $G$ on $L^{2}(G / H)$ such that $\beta$ is an intertwining operator for the $G$-actions.

The paralles to the classical case goes further. In particular one can descibe the image of $\beta$ by a positivity condition: $\beta\left(H_{2}(C)\right)$ is the direct sum of all the holomorphic discrete series from [ÓØ91] which are $C$-admissible. Point evaluation is also continuous and thus defines a kernel, the CauchySzegö kernel $K(\cdot, \cdot)$. This kernel is determined by a holomorphic $H$-invariant function $\Theta_{K}: \Xi\left(C^{o}\right) \rightarrow \mathbb{C}$ such that

$$
K\left(\gamma_{1} \cdot x_{o}, \gamma_{2} \cdot x_{o}\right)=\Theta_{K}\left(\gamma_{2}^{*} \gamma_{1} \cdot x_{o}\right) .
$$

For the first variable fixed, the kernel extends in the second variable smoothly to the boundary of $\Xi\left(C^{o}\right)$. Then the inverse of the boundary 
value map is given just as in the classical case

$$
f(z)=\int_{G / H} \beta(f)(x) K(z, x) d x=\int_{G / H} f(\dot{g}) \Theta_{K}\left(g^{-1} \gamma \cdot x_{o}\right) d \dot{g},
$$

where $z=\gamma \cdot x_{o} \in \Xi\left(C^{o}\right)$ and $\dot{g}=g \cdot x_{o} \in G / H$.

Let $H_{2}(C)=\oplus_{\delta} H_{\delta}$ be the decomposition of $H_{2}(C)$ into holomorphic discrete series. Then each of the representations $\epsilon_{\delta}$ on $H_{\delta}$ give rise to a spherical distribution

$$
\Theta_{\delta}(f):=\operatorname{pr}_{\delta}(f)\left(x_{o}\right)
$$

where $\operatorname{pr}_{\delta}$ is the orthogonal projection onto $H_{\delta}$. The distribution $\Theta_{\delta}$ has an analytic continuation to $\Xi^{o}$, determined up to a constant by a spherical function ([Ó97a, Ó97b, Ó00]), with a well-known expansion formula in terms of elementary functions. On $\Xi\left(C^{o}\right)$ we have then the identity $\sum_{\delta} \Theta_{\delta}=$ $\Theta_{K}$. It is still an open problem to evaluate this sum in general.

To calculate $\Theta_{K}$ independently, in a series of lectures at the University of Poitiers in 1990 by B. Ørsted and one of the authors the problem of relating the Hardy spaces $H_{c l}$ and $H_{2}(C)$ via a causal compactification $G / H \rightarrow \check{S}_{1}$, with $\check{S}_{1}$ the Bergman-Šilov boundary of $G_{1} / K_{1}$, was discussed. It was shown that for the Cayley type spaces those are actually isomorphic (modulo a double covering in some cases) ([ÓØ99]). In particular this result gives a formula for $K(\cdot, \cdot)$ in terms of the well-known classical Cauchy-Szegö kernel. The above summation formula can then be interpreted as a $G$-equivariant decomposition of the classical Cauchy-Szegö kernel or a generalized Heine formula ([ÓØ99]).

Further results in this direction for special cases were obtained by K. Koufany and B. Ørsted ([KØ96, KØ97]), G.I. Ol'shansǩ $([\mathbf{O 9 5}])$ and V.F. Molchanov for $S O(2, n) / S O(1, n)$ ([M97]). The Cayley type spaces were also studied via a Jordan algebra approach by M. Chadli in his thesis ([C96]).

One of the obstacles for obtaining general results in this direction was the lack of general theory for the causal compactification of compactly causal symmetric spaces. This was finally obtained in [B97]. It was shown that there is a natural causal compactification of a "central extension" of most of those spaces in the Bergman-Šilov boundary of the bounded realization of a tube type domain $T_{\Omega}$.

In this paper we extend the results form [B97] to the domain $\Xi\left(C^{o}\right)$ and show that there is a holomorphic function $\psi_{m}: G_{1} / K_{1} \rightarrow \mathbb{C}$ such that

$$
\Xi\left(C^{o}\right)=\left\{z \in G_{1} / K_{1} \mid \psi_{m}(z) \neq 0\right\} .
$$

The map $I$ from the classical Hardy space into $H_{2}(C)$ is then given by $f \mapsto f \sqrt[m]{\psi_{m}}$. In general this has only meaning on a $m$-fold covering of $\Xi\left(C^{o}\right)$. In that case we get an isomorphism into a Hardy space $\widetilde{H}_{2 \text {,odd }}(C)$ on this covering of $\Xi\left(C^{o}\right)$. The image is a direct sum of highest weight moduls. 
We describe the image in terms of the lowest $K$-type of the highest-weight modules.

We remark that similar results to ours can also be obtained with Jordan algebra methods ([Bal $]$ ).

The paper is organized as follows. In the first section we recall some structure theory for causal symmetric spaces and recollect the facts needed by us from [B97] on causal compactifications.

Section 3 is devoted to the description of the Hardy space $H_{2}(C)$ and here we introduce the semigroup $\Gamma$ and the domain $\Xi\left(C^{o}\right)$.

We construct the function $\psi_{m}$ in Section 4. In Section 5 we give an isometry from $L^{2}\left(\check{S}_{1}\right)$ onto $L^{2}(G / H)$, and construct the covering $\widetilde{\Xi}\left(C^{o}\right) \rightarrow$ $\Xi\left(C^{o}\right)$ of $\Xi\left(C^{o}\right)$ corresponding to the $m$-th root of $\psi_{m}$.

In Section 6 we show that that $\Xi\left(C^{o}\right)=\left\{z \in G_{1} / K_{1} \mid \psi_{m}(z) \neq 0\right\}$. This gives us the necessary tool to analyze the covering $\widetilde{\Xi}\left(C^{o}\right)$ in more detail in the next section. In particular we show that we need at most a double covering for decribing the Hardy spaces. The final result of this paper is Theorem 7.8 where we characterize the image of $I$.

The authors would like to thank the Institute Mittag-Leffler for their hospitality during their stay there in the Spring of 1996. The first author would like to thank Louisiana State University and the second author for their hospitality during his stay in September, 1996.

\section{Causal compactifications.}

In this section we collect some standard facts on symmetric spaces. We also collect some newer results on causal compactifications. We use the monograph [HÓ96] and the orginal papers [ÓØ88, Ó91, B97] as references. We call $(G, H, \tau)$ a symmetric space, when $G$ is a connected Lie group, $\tau: G \rightarrow G$ is an involutive automorphism, and $H \subset G$ a closed subgroup with

$$
\left(G^{\tau}\right)_{o} \subset H \subset G^{\tau}
$$

where $G^{\tau}:=\{a \in G \mid \tau(a)=a\}$ and the subscript o denotes the connected component containing the identity. By abuse of notation we then also call $G / H$ a symmetric space. A symmetric Lie algebra is a triple $(\mathfrak{g}, \mathfrak{h}, \tau)$, where $\mathfrak{g}$ is a Lie algebra, $\tau: \mathfrak{g} \rightarrow \mathfrak{g}$ is an involutive automorphism, and $\mathfrak{h}=\{X \in \mathfrak{g} \mid \tau(X)=X\}$ the subalgebra of $\tau$-fixed elements. To every symmetric space $(G, H, \tau)$ there is associated the symmetric algebra $\left(\mathfrak{g}, \mathfrak{h}, \tau^{\prime}\right):=(\operatorname{Lie}(G), \operatorname{Lie}(H), d \tau)$. In the sequel we will denote the differential of $\tau$ (and similarly the differentials of all other group homomorphisms) always by the same letter. The symmetric algebra $(\mathfrak{g}, \mathfrak{h}, \tau)$ is irreducible if there is no nontrivial $\tau$-stable ideal of $\mathfrak{g}$ not contained in $\mathfrak{h}$. The symmetric algebra $(\mathfrak{g}, \mathfrak{h}, \tau)$ is called reductive respectively semisimple if $\mathfrak{g}$ is reductive respectively semisimple. 
Let $(\mathfrak{g}, \mathfrak{h}, \tau)$ be a reductive symmetric algebra. Choose a Cartan involution $\theta$ of $\mathfrak{g}$ commuting with $\tau$ ([H78, p. 192] or [Ó84, Lemma 2.1]). Let $\mathfrak{q}:=$ $\{X \in \mathfrak{g} \mid \tau(X)=-X\}, \mathfrak{k}:=\{X \in \mathfrak{g} \mid \theta(X)=X\}$, and $\mathfrak{p}:=\{X \in \mathfrak{g} \mid$ $\theta(X)=-X\}$, then

$$
\mathfrak{g}=\mathfrak{h} \oplus \mathfrak{q}=\mathfrak{k} \oplus \mathfrak{p}=\mathfrak{h}_{k} \oplus \mathfrak{h}_{p} \oplus \mathfrak{q}_{k} \oplus \mathfrak{q}_{p},
$$

where an index denotes the intersection with the corresponding subspace, i.e., $\mathfrak{h}_{p}:=\mathfrak{h} \cap \mathfrak{p}$, etc.

Let $\mathfrak{g}_{s}:=[\mathfrak{g}, \mathfrak{g}]$ denote the semisimple part of $\mathfrak{g}$ and $\mathfrak{z}$ the center of $\mathfrak{g}$. If $\mathfrak{l}$ is a subspace of $\mathfrak{g}$ such that $\mathfrak{l}=\mathfrak{l} \cap \mathfrak{z} \oplus \mathfrak{l} \cap \mathfrak{g}_{s}$, then we set $\mathfrak{l}_{z}:=\mathfrak{l} \cap \mathfrak{z}$, and $\mathfrak{l}_{s}:=\mathfrak{l} \cap \mathfrak{g}_{s}$. For $(G, H, \tau)$ associated to $(\mathfrak{g}, \mathfrak{h}, \tau)$, let $G_{s}$ be the analytic subgroup corresponding to $\mathfrak{g}_{s}$, and $Z:=\exp \mathfrak{z}$ those corresponding to $\mathfrak{z}$. Then $G=Z G_{s}$ and $D:=Z \cap G_{s}$ is a discrete central subgroup in $G_{s}$. Moreover we have a (right) action

$$
D \times\left(Z \times G_{s}\right) \rightarrow Z \times G_{s}, \quad(d,(z, g)) \mapsto\left(z d, d^{-1} g\right),
$$

such that

$$
G \simeq\left(Z \times G_{s}\right) / D=: Z \times{ }_{D} G_{s} .
$$

Let $E$ be a finite dimensional vector space over the reals. A subset $C \subset E$ is called a cone if $C$ is convex and closed under multiplication by $\mathbb{R}^{+}$. The closed cone $C$ is pointed if $C \cap-C=\{0\}$ and generating if $C-C=E$. A pointed generating cones is called regular. We remark that a cone $C$ is generating if and only if its interior $C^{o}$ is nonempty.

Definition. Assume that $(G, H, \tau)$ is a symmetric space with associated reductive symmetric Lie algebra $(\mathfrak{g}, \mathfrak{h}, \tau)$.

1) The symmetric space is called compactly causal if there exists a $\mathrm{H}$ invariant regular cone $C$ in $\mathfrak{q}$ such that $C^{o} \cap \mathfrak{k} \neq \emptyset$.

2) $(\mathfrak{g}, \mathfrak{h}, \tau)$ is called compactly causal if $\left(G,\left(G^{\tau}\right)_{o}, \tau\right)$ is compactly causal.

3) The symmetric space is called noncompactly causal if there exists a $H$-invariant regular cone $C \subset \mathfrak{q}$ such that $C^{o} \cap \mathfrak{p} \neq \emptyset$.

4) $(G, H, \tau)$ is called of Cayley type if it is semisimple and both compactly and noncompactly causal.

5) Let $\mathfrak{c}\left(\mathfrak{q}_{k}\right):=\left\{X \in \mathfrak{q}_{k} \mid\left[\mathfrak{q}_{k}, X\right]=\{0\}\right\}$. The symmetric space respectively the associated algebra are called of weakly Hermitian type if $\mathfrak{z}_{\mathfrak{q}}\left(\mathfrak{c}\left(\mathfrak{q}_{k}\right)\right)=\mathfrak{q}_{k}$.

Notice that in this definition we do not assume that $G$ is noncompact. Thus every compact symmetric space is weakly Hermitian. On the other hand a compact symmetric space can only be compactly causal if it is reductive ([HÓ96]). The spaces we consider will by construction be both compactly causal and of weakly Hermitian type. We refer to [KN96] and [KNÓ97] for a general discussion of the connection between weakly Hermitian and compactly causal. 
Let $\left(\mathfrak{g}_{1}, \mathfrak{h}_{1}, \tau_{1}\right)$ be a compactly causal irreducible symmetric algebra with $\mathfrak{g}_{1}$ simple and noncompact. Let $G_{1 \mathbb{C}}$ the simply connected complex Lie group with Lie algebra $\mathfrak{g}_{1 \mathbb{C}}:=\mathfrak{g}_{1} \otimes \mathbb{C}$. We extend $\tau_{1}$ to a complex linear involution of $\mathfrak{g}_{1 \mathbb{C}}$, and denote this involution and the corresponding involution on $G_{1 \mathbb{C}}$ again by $\tau_{1}$. Then, as $G_{1 \mathbb{C}}$ is assumed simply connected, it follows that $H_{1 \mathbb{C}}:=G_{1 \mathbb{C}}^{\tau_{1}}$ is connected ([L69, p. 171]). Let $G_{1}$ be the analytic subgroup of $G_{1 \mathbb{C}}$ with Lie algebra $\mathfrak{g}_{1}$ and

$$
H_{1}:=G_{1} \cap H_{1 \mathbb{C}}=G_{1}^{\tau_{1}} .
$$

Let $\theta_{1}$ be a Cartan involution commuting with $\tau_{1}$, and let $\mathfrak{g}_{1}=\mathfrak{k}_{1} \oplus \mathfrak{p}_{1}$ be the corresponding Cartan decomposition. Recall that for $\left(\mathfrak{g}_{1}, \mathfrak{h}_{1}, \tau_{1}\right)$ irreducible compactly causal the Riemannian symmetric space $G_{1} / K_{1}$ is Hermitian symmetric ([HÓ96, Remark 3.19]) and that $\left(\mathfrak{g}_{1}, \mathfrak{h}_{1}, \tau_{1}\right)$ is of weakly Hermitian type ([HÓ96, Lemma 1.2.1, Lemma 1.3.5]). We will assume that $G_{1} / K_{1}$ is a tube type domain.

Let $\mathfrak{t}_{1}$ be a Cartan subalgebra of $\mathfrak{k}_{1}$. Let $\Delta\left(\mathfrak{g}_{1 \mathbb{C}}, \mathfrak{t}_{1 \mathbb{C}}\right)$ be the set of roots of $\mathfrak{t}_{1 \mathbb{C}}$ in $\mathfrak{g}_{1 \mathbb{C}}$. We have the two subsets

$$
\Delta\left(\mathfrak{k}_{1 \mathbb{C}}, \mathfrak{t}_{1 \mathbb{C}}\right)=\left\{\alpha \in \Delta\left(\mathfrak{g}_{1 \mathbb{C}}, \mathfrak{t}_{1 \mathbb{C}}\right) \mid \mathfrak{g}_{1 \mathbb{C}, \alpha} \subset \mathfrak{k}_{1 \mathbb{C}}\right\}
$$

of compact and

$$
\Delta\left(\mathfrak{p}_{1 \mathbb{C}}, \mathfrak{t}_{1 \mathbb{C}}\right)=\left\{\alpha \in \Delta\left(\mathfrak{g}_{1 \mathbb{C}}, \mathfrak{t}_{1 \mathbb{C}}\right) \mid \mathfrak{g}_{1 \mathbb{C}, \alpha} \subset \mathfrak{p}_{1 \mathbb{C}}\right\}
$$

of noncompact roots. We choose an ordering in $i t_{1}^{*}$ such that the positive noncompact dominate the positive compact roots and define

$$
\mathfrak{p}_{1}^{+}:=\sum_{\alpha \in \Delta^{+}\left(\mathfrak{p}_{1 \mathbb{C}}, \mathfrak{t}_{\mathbb{C}}\right)} \mathfrak{g}_{1 \mathbb{C}, \alpha} \text { respectively } \mathfrak{p}_{1}^{-}:=\sum_{\alpha \in \Delta^{+}\left(\mathfrak{p}_{1 \mathbb{C}}, \mathfrak{t}_{1 \mathbb{C}}\right)} \mathfrak{g}_{1 \mathbb{C},-\alpha} .
$$

Recall that two roots $\alpha$ and $\beta$ are strongly orthogonal if $\alpha \neq \pm \beta$ and $\alpha \pm \beta \notin \Delta\left(\mathfrak{g}_{1 \mathbb{C}}, \mathfrak{t}_{1 \mathbb{C}}\right)$. Let $\left\{\gamma_{1}, \ldots, \gamma_{r}\right\} \subset \Delta^{+}\left(\mathfrak{p}_{1 \mathbb{C}}, \mathfrak{t}_{1 \mathbb{C}}\right)$ be a maximal system of strongly orthogonal roots. Let $H_{j} \in\left[\mathfrak{g}_{1 \mathbb{C}, \gamma_{j}}, \mathfrak{g}_{1 \mathbb{C},-\gamma_{j}}\right] \cap i \mathfrak{t}_{1}$ be such that $\gamma_{i}\left(H_{j}\right)=2 \delta_{i, j}$. Choose $E_{ \pm j} \in \mathfrak{g}_{1 \mathbb{C}, \pm \gamma_{j}}$ such that

$$
X_{j}:=E_{j}+E_{-j}, Y_{j}:=i E_{j}-i E_{-j} \in \mathfrak{p}_{1},
$$

and

$$
\left[E_{j}, E_{-j}\right]=H_{j}
$$

It is known that $\mathfrak{a}_{p}:=\sum \mathbb{R} X_{j}$ is maximal abelian in $\mathfrak{p}_{1}([\mathbf{H 7 8}, \mathrm{p} .387])$. Let

$$
X^{0}:=\sum X_{j}, \quad Y^{0}:=\sum Y_{j}, \quad \text { and } \quad Z^{0}:=-\frac{1}{2} \sum i H_{j},
$$

then $Z^{0} \in \mathfrak{z}\left(\mathfrak{k}_{1}\right)$, with $\mathfrak{z}\left(\mathfrak{k}_{1}\right)$ the center of $\mathfrak{k}_{1}$, and ad $X^{0}$ has eigenvalues 0,2 and -2 . Let

$$
\eta:=\operatorname{Ad}\left(\exp \frac{\pi}{2} i X^{0}\right)
$$


be the involution on $G_{1 \mathbb{C}}$ respectively $\mathfrak{g}_{1 \mathbb{C}}$ given by conjugation with $\exp \frac{\pi}{2} i X^{0}$. Then the symmetric space $G_{1} / G_{1}^{\eta}$ is of Cayley type and

$$
\mathfrak{q}_{1 \eta}:=\mathfrak{g}_{1}^{-\eta}=\left\{X \in \mathfrak{g}_{1} \mid \eta(X)=-X\right\}
$$

is the direct sum of the two eigenspaces $\mathfrak{q}_{1 \eta}^{ \pm}:=\left\{X \in \mathfrak{q}_{1 \eta} \mid\left[X^{0}, X\right]= \pm 2 X\right\}$ of ad $X^{0}$, which are also $G_{1}^{\eta}$-invariant. As $\theta_{1}\left(X^{0}\right)=-X^{0}$ we get $\theta_{1}\left(\mathfrak{q}_{1 \eta}^{+}\right)=\mathfrak{q}_{1 \eta}^{-}$.

Define $Y_{ \pm} \in \mathfrak{q}_{1 \eta}^{ \pm}$by $Y^{0}=Y_{+}+Y_{-}$. The sets $C_{ \pm}:=\overline{\operatorname{Ad}\left(G_{1}^{\eta}\right) Y_{ \pm}}$are regular cones in $\mathfrak{q}_{1 \eta}^{ \pm}$and the cone $C_{k}:=C_{+}-C_{-} \subset \mathfrak{q}_{1 \eta}$ defines the compactly causal structure on $G_{1} / G_{1}^{\eta}$. Let $\mathfrak{p}^{\prime}$ be the sum of the 0 - and (-2)-eigenspaces of ad $X^{0}$. Then $P^{\prime}:=N_{G_{1}}\left(\mathfrak{p}^{\prime}\right)$ is a parabolic subgroup and

$$
\check{S}_{1}:=G_{1} / P^{\prime}=K_{1} / K_{1}^{\eta}
$$

is the Bergman-Šilov boundary of $G_{1} / K_{1}$. The compact symmetric space $\check{S}_{1}$ is causal with causal structure defined by $C_{+} \subset \mathfrak{q}_{1 \eta}^{+} \simeq T_{e P^{\prime}} \check{S}_{1}$. Moreover

$$
G_{1}^{\eta}=Z_{G_{1}}\left(X^{0}\right) \subset P^{\prime},
$$

and the canonical projection $\Phi_{1}: G_{1} / G_{1}^{\eta} \rightarrow \check{S}_{1}$ is causal.

Under conditions specified in [B97] there exists an involution $\sigma$, commuting with $\tau_{1}, \theta_{1}$, and $\eta$, such that

$$
(G, H, \tau):=\left(\left(G_{1}^{\sigma}\right)_{o}, H_{1} \cap\left(G_{1}^{\sigma}\right)_{o}, \tau_{1} \mid\left(G_{1}^{\sigma}\right)_{o}\right)
$$

is a compactly causal symmetric subspace of $\left(G_{1}, G_{1}^{\eta}, \eta\right)$ and $\Phi_{1}$ can be used to define a causal compactification of it. (For spaces of Cayley type $\left(\mathfrak{g}_{1}, \mathfrak{h}_{1}, \tau_{1}\right)$ in the preceding discussion has to be replaced by $\left(\mathfrak{g}_{1} \times \mathfrak{g}_{1}, \mathfrak{h}_{1} \times\right.$ $\left.\mathfrak{h}_{1}, \tau_{1} \times \tau_{1}\right)$ and $\sigma(g, h)=(h, g)$.) For the general theory we will not need the explicit form of $\sigma$ but only the "axiomatic" properties of the construction that we collect in the remaining part of this section.

Define $\theta=\left.\theta_{1}\right|_{G}$ then $\theta$ is a Cartan involution on $G$ commuting with $\tau$. We denote the corresponding Cartan decomposition by $\mathfrak{g}=\mathfrak{k} \oplus \mathfrak{p}$. Let $\mathfrak{g}_{1}=\mathfrak{g}+\mathfrak{q}_{1 \sigma}$ be the eigenspace decomposition of $\sigma$. We have $\left.\eta\right|_{G}=\left.\tau_{1}\right|_{G}$ - in fact, in many cases $\eta=\tau_{1}$ - such that $G \cap G_{1}^{\eta}=G \cap H_{1}$ and also $\mathfrak{q}:=\mathfrak{q}_{1} \cap \mathfrak{g}=\mathfrak{q}_{1 \eta} \cap \mathfrak{g}$.

Lemma 2.1. With notation as above the following holds:

(1) The algebra $\mathfrak{b}:=\mathfrak{a}_{p} \cap \mathfrak{q}_{1 \sigma}$ is maximal abelian in $\mathfrak{p}_{1} \cap \mathfrak{q}_{1 \sigma}, X^{0} \in \mathfrak{b}$, $Y^{0} \in \mathfrak{p}_{1} \cap \mathfrak{q}_{1 \sigma}$, and $Z^{0} \in \mathfrak{k} \cap \mathfrak{q}$.

(2) The cone $C_{k}$ is $\sigma$-invariant.

(3) Let $\mathrm{pr}_{\mathfrak{q}}: \mathfrak{q}_{1 \eta} \rightarrow \mathfrak{q}$ be the projection with respect to the decomposition $\mathfrak{q}_{1 \eta}=\mathfrak{q} \oplus \mathfrak{q}_{1 \eta} \cap \mathfrak{q}_{1 \sigma}$. Then $C_{k} \cap \mathfrak{q}=\operatorname{pr}_{\mathfrak{q}}\left(C_{k}\right)$ and $\left(\operatorname{pr}_{\mathfrak{q}}\left(C_{k}\right)\right)^{o} \cap \mathfrak{k} \neq \emptyset$.

Proof. (1) With $Z^{0}=\frac{1}{4}\left[X^{0}, Y^{0}\right]$ the last claim follows from the preceding ones. These are prerequisites for the construction of $\sigma$ in $[\mathbf{B 9 7}$, Theorem 5.1] respectively [B97, Theorem 5.9]. 
(2) From

$$
Y_{+}+Y_{-}=Y^{0}=-\sigma\left(Y^{0}\right)=-\sigma\left(Y_{+}\right)-\sigma\left(Y_{-}\right)
$$

and

$$
\sigma\left(Y_{ \pm}\right)=\sigma\left( \pm \frac{1}{2}\left[X^{0}, Y_{ \pm}\right]\right)=\mp \frac{1}{2}\left[X^{0}, \sigma\left(Y_{ \pm}\right)\right]
$$

we have $\sigma\left(Y_{ \pm}\right)=-Y_{\mp}$. By the $\sigma$-invariance of $G_{1}^{\eta}$ this implies the claim together with the definition of $C_{k}$.

(3) The $\sigma$-invariance of $C_{k}$ implies that $C_{k} \cap \mathfrak{q}=\operatorname{pr}_{\mathfrak{q}}\left(C_{k}\right)$. That $\left(\operatorname{pr}_{\mathfrak{q}}\left(C_{k}\right)\right)^{o} \cap$ $\mathfrak{k}$ is nonempty follows since $Z^{0} \in C_{k}^{o}$.

By the last part of the Lemma $C:=C_{k} \cap \mathfrak{q}$ is a $H$-invariant regular cone in $\mathfrak{q}$ which defines a compactly causal structure for $(G, H, \tau)$. It is clear that this definition makes $(G, H, \tau)$ into a causal subspace of $\left(G_{1}, G_{1}^{\eta}, \eta\right)$.

Corollary 2.2. $C+\mathfrak{p}^{\prime}=C_{k}+\mathfrak{p}^{\prime}=C_{+} \oplus \mathfrak{p}^{\prime}$.

Proof. By definition we have

$$
C+\mathfrak{p}^{\prime} \subset C+\mathfrak{p}^{\prime}=C_{+} \oplus \mathfrak{p}^{\prime} .
$$

When we write $X \in C_{+}$in the form $(X+\sigma(X))-\sigma(X)$, where $X+\sigma(X) \in$ $C_{k} \cap \mathfrak{g}$ and $\sigma(X) \in \sigma\left(\mathfrak{q}_{1 \eta}^{+}\right) \subset \mathfrak{p}^{\prime}$, the other inclusion is also obvious.

Definition ([ÓØ99]). Let $M$ and $N$ be manifolds, with $N$ compact, and with causal structure $M \ni \mapsto C(m) \subset T_{m} M$ respectively $N \ni n \mapsto D(n) \subset$ $T_{n} N$. Let $\Phi: M \rightarrow N$ be smooth. The pair $(N, \Phi)$ is called a causal compactification of $M$ if $\Phi$ is a diffeomorphism onto an open dense subset of $N$, and $(d \Phi)_{m}(C(m))=D(\Phi(m))$ for all $m \in M$. Let $L$ be a Lie group acting on $M$ and $N$ such that the causal structures are $L$-invariant. Then the causal compactification is $L$-equivariant if $\Phi$ is $L$-equivariant.

Theorem 2.3. The canonical inclusion $\iota: G / H \hookrightarrow G_{1} / G_{1}^{\eta}$ is causal and the map $\Phi:=\Phi_{1} \circ \iota: G / H \hookrightarrow \check{S}_{1}$ is a $G$-equivariant causal compactification of $G / H$.

Proof. By [B97] we know that $\Phi: G / H \rightarrow \check{S}_{1}$ is an injective $G$-map with open and dense image. With the canonical identifications $T_{e H} G / H \simeq \mathfrak{q}$,

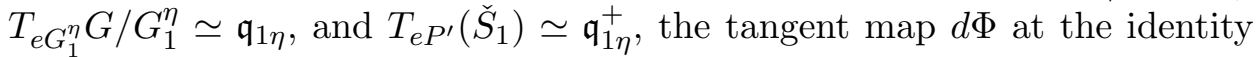
coset is given by first imbedding $\mathfrak{q}$ into $\mathfrak{q}_{1 \eta}$ and then projecting onto $\mathfrak{q}_{1 \eta}^{+}$. Thus $d \Phi_{e H}(C)=C_{+}$by Corollary 2.2. By the $G$-invariance of the causal structures on $G / H$ respectively $\breve{S}_{1}$ this is all to show.

As all groups are subgroups of $G_{1 \mathbb{C}}$ it is immediate to see that $\Phi$ extends

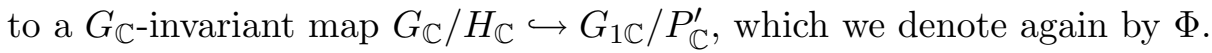


Example. Let $G_{1}=S U(1,1)$. Denote by $E_{i, j}$ the matrix with entry 1 in the $i$-th row and $j$-th column and otherwise zero. We choose $E_{1}=E_{1,2}$, $E_{-1}=E_{2,1}$, and $H_{1}=E_{1,1}-E_{2,2}$. Then

$$
X^{0}=\left(\begin{array}{ll}
0 & 1 \\
1 & 0
\end{array}\right), \quad \text { and } \quad Y^{0}=\left(\begin{array}{cc}
0 & i \\
-i & 0
\end{array}\right) .
$$

Thus $\eta$ is given by conjugation by $i X^{0}$. This is easily seen to be the same involution on $S U(1,1)$ as $g \mapsto \bar{g}$, the complex conjugation. We will also need the Cayley transform, which is given by conjugation by

$$
c:=\exp \frac{\pi}{4} i Y^{0}=\frac{1}{\sqrt{2}}\left(\begin{array}{cc}
1 & -1 \\
1 & 1
\end{array}\right) .
$$

As is well-known, the unit disk - identifying $\mathbb{C} \simeq \mathbb{C} E_{1}=\mathfrak{p}_{1}^{+}$- is the Harish-Chandra realization of $G_{1} / K_{1}$, where $G_{1}$ operates by

$$
\left(\begin{array}{ll}
a & b \\
c & d
\end{array}\right) \cdot z=\frac{a z+b}{c z+d}, \quad\left(\begin{array}{ll}
a & b \\
c & d
\end{array}\right) \in S U(1,1) .
$$

The Bergman-Šilov boundary $\check{S}_{1}$ is now the unit circle and the Cayley transform $z \mapsto c \cdot z$ maps the origin to $-1 \in \check{S}_{1}$. We notice that $\operatorname{Ad}(c) H_{1}=X^{0}$. It follows in particular that $\left(\operatorname{Ad}(c)\left(\mathfrak{k}_{1 \mathbb{C}}+\mathfrak{p}_{1}^{-}\right)\right) \cap \mathfrak{g}_{1}=\mathfrak{p}^{\prime}$ and $\check{S}_{1}=G_{1} / P^{\prime} \simeq$ $G_{1} \cdot(-1)$. With $\check{S}_{1}$ in the Harish-Chandra realization the map $\Phi_{1}$ is therefore given by $g G_{1}^{\eta} \mapsto g \cdot(-1)$.

We can choose the involution $\sigma=\theta_{1}$, i.e.,

$$
(G, H)=\left(\left\{\left(\begin{array}{cc}
a & 0 \\
0 & \bar{a}
\end{array}\right) \mid a \in S^{1}\right\},\{ \pm \mathrm{id}\}\right),
$$

and then

$$
\Phi\left(\left(\begin{array}{cc}
a & 0 \\
0 & \bar{a}
\end{array}\right) H\right)=-a^{2} .
$$

We restrict us in the following to causal compactifications constructed as in the Theorem. (Inded, as is shown in [B97], with one natural additional assumption all causal compactifications in the Bergman-Šilov boundary are of this form.) A list of the possible compactifications can be found in [B97], cf. also below.

For short we call $\langle A, B, C\rangle$ a $\mathfrak{s l}(2)$-triple if

$$
\left(\begin{array}{cc}
1 & 0 \\
0 & -1
\end{array}\right) \mapsto A,\left(\begin{array}{ll}
0 & 1 \\
0 & 0
\end{array}\right) \mapsto B, \text { and }\left(\begin{array}{cc}
0 & 0 \\
1 & 0
\end{array}\right) \mapsto C
$$

defines an isomorphism from $\mathfrak{s l}(2, \mathbb{C})$ onto the complex Lie algebra generated by $\{A, B, C\}$. 
Proposition 2.4. Let the notation be as above. Then the following holds:

(1) There exist $\lambda_{j^{\prime}, j} \in\{0,1\}$ such that with

$$
\begin{gathered}
E_{ \pm j^{\prime}}^{\prime}:=\sum_{j} \lambda_{j^{\prime}, j} E_{ \pm j}, \\
H_{j^{\prime}}^{\prime}:=\left[E_{j^{\prime}}^{\prime}, E_{-j^{\prime}}^{\prime}\right]=\sum_{j} \lambda_{j^{\prime}, j}^{2} H_{j}
\end{gathered}
$$

and

$$
X_{j^{\prime}}^{\prime}:=E_{j^{\prime}}^{\prime}+E_{-j^{\prime}}^{\prime}=\sum_{j} \lambda_{j^{\prime}, j} X_{j}
$$

we have $\mathfrak{b}=\sum \mathbb{R} X_{j^{\prime}}^{\prime}$, and the triples $\left\langle H_{j^{\prime}}^{\prime}, E_{j^{\prime}}^{\prime}, E_{-j^{\prime}}^{\prime}\right\rangle$ are pairwise commuting $\mathfrak{s l}(2)$-triples.

(2) With $c:=\exp \left(\frac{\pi}{4} i Y^{0}\right)$, the Cayley-transformed space $\mathfrak{a}:=\operatorname{Ad}(c) i \mathfrak{b}$ is maximal abelian in $\mathfrak{k} \cap \mathfrak{q}$.

(3) Let $\mathfrak{t}_{1}^{-}:=\sum \mathbb{R} i H_{j}$ then we have $\mathfrak{t}_{1}^{-}=\mathfrak{a}+\mathfrak{t}_{1}^{-} \cap \mathfrak{q}_{1 \sigma}$.

(4) We have $\mathfrak{z} \subset \mathfrak{k}$ and, if $\mathfrak{g}$ is noncompact, then $(\mathfrak{g}, \mathfrak{h}, \tau)$, is weakly Hermitian. If $\mathfrak{l} \subset \mathfrak{h}$ is an ideal of $\mathfrak{g}$ then $\mathfrak{l} \subset \mathfrak{k}$.

(5) $C^{o}=\operatorname{Ad}(H)\left(\mathfrak{a} \cap C^{o}\right)$ and $C=\operatorname{Ad}(H)(\mathfrak{a} \cap C)$.

(6) $\mathfrak{a} \cap C^{o}=-\sum \mathbb{R}^{+} i H_{j^{\prime}}^{\prime}$ and $\mathfrak{a} \cap C=-\sum \mathbb{R}_{0}^{+} i H_{j^{\prime}}^{\prime}$.

Proof. (1) This is a direct consequence of [B97, Theorem 5.1] respectively [B97, Theorem 5.9].

(2) The inner automorphism $\operatorname{Ad}(c)$ maps the subalgebra

$$
\mathfrak{g}_{a}:=\mathfrak{k}+i\left(\mathfrak{p}_{1} \cap \mathfrak{q}_{1 \sigma}\right)
$$

onto itself, since $Y^{0} \in \mathfrak{p}_{1} \cap \mathfrak{q}_{1 \sigma}$ by Lemma 2.1 .

We consider first the causal compactifications described by [B97, Theorem 5.1]. Here we have $\tau_{1}=\eta$ and $\mathfrak{b} \subset \mathfrak{h}_{1}$. Then $Y^{0}=\left[X^{0}, Z^{0}\right] \in \mathfrak{p}_{1} \cap \mathfrak{q}_{1}$ is $\tau_{1} \theta_{1}$-fixed and consequently $\mathfrak{k} \cap \mathfrak{q}+i\left(\mathfrak{p}_{1} \cap \mathfrak{q}_{1 \sigma} \cap \mathfrak{h}_{1}\right)$, the $(-1)$-eigenspace of the involution $\tau_{1} \theta_{1}$ in $\mathfrak{g}_{a}$, is $\operatorname{Ad}(c)$-invariant. Using $\theta_{1}=\operatorname{Ad}\left(\exp \pi Z^{0}\right)$, by a $\mathfrak{s l}(2)$-calculation $\operatorname{Ad}(c) \theta_{1}=\tau_{1} \operatorname{Ad}(c)$. Looking at the corresponding eigenspaces in $\mathfrak{k} \cap \mathfrak{q}+i\left(\mathfrak{p}_{1} \cap \mathfrak{q}_{1 \sigma} \cap \mathfrak{h}_{1}\right)$ we get $\operatorname{Ad}(c) i\left(\mathfrak{p}_{1} \cap \mathfrak{q}_{1 \sigma} \cap \mathfrak{h}_{1}\right)=\mathfrak{k} \cap \mathfrak{q}$. As $\mathfrak{b} \subset \mathfrak{p}_{1} \cap \mathfrak{q}_{1 \sigma} \cap \mathfrak{h}_{1}$, the claim for this case then follows.

For the compactifications given by [B97, Theorem 5.9] we have $\mathfrak{b} \subset \mathfrak{q}_{1}$. Similarly to the first case we get successively $Y^{0} \in \mathfrak{h}_{1}$, the $\operatorname{Ad}(c)$-invariance of $\mathfrak{k} \cap \mathfrak{q}+i\left(\mathfrak{p}_{1} \cap \mathfrak{q}_{1 \sigma} \cap \mathfrak{q}_{1}\right)$, and $\operatorname{Ad}(c) \theta_{1} \tau_{1}=\sigma \operatorname{Ad}(c)$, where $\sigma:=\eta \circ \tau_{1}$ as in $[\mathbf{B 9 7}$, Thm. 5.9] defined. Therefore $\operatorname{Ad}(c)$ maps the $(+1)$-eigenspace of $\theta_{1} \tau_{1}$ onto the $(+1)$-eigenspace of $\sigma$, i.e., $\operatorname{Ad}(c) i\left(\mathfrak{p}_{1} \cap \mathfrak{q}_{1 \sigma} \cap \mathfrak{q}_{1}\right)=\mathfrak{k} \cap \mathfrak{q}$. Now the stated result for this case follows again from Lemma 2.1.

(3) For the orthogonal sum $\mathfrak{a}_{p}=\mathfrak{b} \oplus \mathfrak{b}^{\perp}$ (with respect to the inner product $(X, Y)=-B(X, \theta(Y)))$ we have by Lemma 2.1 that $\mathfrak{b}^{\perp} \subset \mathfrak{p}_{1} \cap \mathfrak{g}$. Now $\mathfrak{t}_{1}^{-}=\operatorname{Ad}(c) i \mathfrak{a}_{p}=\mathfrak{a} \oplus \operatorname{Ad}(c) \mathfrak{b}^{\perp}$. The second summand is contained in $\mathfrak{k}_{1}$, since $Z^{0} \in \mathfrak{a}$. But the Cayley transform maps with $\mathfrak{g}_{a}=\mathfrak{k}+i\left(\mathfrak{p}_{1} \cap \mathfrak{q}_{1 \sigma}\right)$ also 
the orthocomplement $\mathfrak{g}_{a}^{\perp}$ of $\mathfrak{g}_{a}$ in $\mathfrak{k}_{1}+i \mathfrak{p}_{1}$ onto itself. With $i \mathfrak{b}^{\perp} \subset \mathfrak{g}_{a}^{\perp}$ we have therefore $\operatorname{Ad}(c) i \mathfrak{b}^{\perp} \subset \mathfrak{g}_{a}^{\perp} \cap \mathfrak{k}_{1}=\mathfrak{k}_{1} \cap \mathfrak{q}_{1 \sigma}$.

(4) As $Z^{0} \in \mathfrak{g}$ by Lemma 2.1, and $\operatorname{ad} Z^{0} \mid \mathfrak{p}_{1}$ is regular, it follows that $\mathfrak{z} \mathfrak{g}\left(Z^{0}\right)=\mathfrak{k}$. Hence

$$
\mathfrak{z} \subset \mathfrak{z} \mathfrak{g}\left(Z^{0}\right)=\mathfrak{k} .
$$

Write $Z^{0}=Z_{1}+Z_{2}$, with $Z_{1} \in \mathfrak{z}(\mathfrak{g})$, and $Z_{2} \in[\mathfrak{g}, \mathfrak{g}]$. Assume that $Z_{2}=0$. Then the same argument shows that $\mathfrak{g}=\mathfrak{z}_{\mathfrak{g}}\left(Z^{0}\right)=\mathfrak{k}$. Hence $\mathfrak{g}$ is compact. Thus, if $\mathfrak{g}$ is not compact it follows that $Z_{2} \neq 0$. Furthermore $\mathfrak{z}_{\mathfrak{g}}\left(Z_{2}\right)=\mathfrak{k}$, as $Z_{1}$ commutes with $\mathfrak{g}$. In particular it follows that $Z_{2} \in \mathfrak{c}\left(\mathfrak{q}_{k}\right)$ and therefore

$$
\mathfrak{z} \mathfrak{q}\left(\mathfrak{c}\left(\mathfrak{q}_{k}\right)\right) \subset \mathfrak{z}_{\mathfrak{q}}\left(Z_{2}\right)=\mathfrak{q}_{k} .
$$

Suppose that $\mathfrak{l}$ is an ideal in $\mathfrak{g}$ contained in $\mathfrak{h}$. As $Z_{2} \in \mathfrak{q}$ it follows that $\left[Z_{2}, \mathfrak{l}\right]=\{0\}$. Hence

$$
\mathfrak{l} \subset \mathfrak{z} \mathfrak{g}\left(Z_{2}\right)=\mathfrak{k}
$$

which shows that $\mathfrak{l}$ is compact.

(5) Let $X \in C^{o}=\left(C_{k}^{o} \cap \mathfrak{g}\right)$. Then $G^{X}=\{a \in G \mid \operatorname{Ad}(a) X=X\}$ is compact. Therefore the elements in $\mathfrak{g}^{X}$ are semisimple and with imaginary eigenvalues (see the proof of [HÓ96, Thm. 4.2.15]). Hence there is a $h \in H$ such that $\operatorname{Ad}(h) X \in \mathfrak{q}_{k}\left(\left[\mathbf{M 7 9}\right.\right.$, Thm. 1]). Let $\mathfrak{a}_{q}$ be a maximal abelian algebra in $\mathfrak{q}_{k}$ containing $X$, then (by [M79, Lemma 7]) we find $k \in H \cap K$ with $\operatorname{Ad}(k) \mathfrak{a}_{q}=\mathfrak{a}$. In particular $\operatorname{Ad}(k h) X \in \mathfrak{a}$. Therefore $C^{o} \subset \operatorname{Ad}(H)(\mathfrak{a} \cap$ $\left.C^{o}\right)$. As the other inclusion is obvious we get $C^{o}=\operatorname{Ad}(H)\left(\mathfrak{a} \cap C^{o}\right)$. Since the eigenvalues of ad $X$ are continuous in $X$, the same argument apply to $X$ in the boundary of $C$. Thus the assertion for the whole cone follows.

(6) By [ÓØ99, Lemma 4.4] or [HÓ96, Chap. 4.2] we have $\mathfrak{t}_{1}^{-} \cap C_{k}^{o}=$ $-\sum \mathbb{R}^{+} i H_{j}$ respectively $\mathfrak{t}_{1}^{-} \cap C_{k}=-\sum \mathbb{R}_{0}^{+} i H_{j}$. (Note that we have here a minus sign by our choice of $Z^{0}$.) The claims then follow with $\mathfrak{t}_{1}^{-} \cap \mathfrak{a}=$ $\sum \mathbb{R} i H_{j^{\prime}}^{\prime}$

Remarks. (1) The $\lambda_{j^{\prime}, j}$ are known by [B97, Thm. 5.1, Thm. 5.9] for every case. Either $\lambda_{j^{\prime}, j}=\delta_{j^{\prime}, j}$, for $j^{\prime}=1, \ldots, r$, or $r=2 r^{\prime}$ and $\lambda_{j^{\prime}, j}=\delta_{j^{\prime}, j}+\delta_{j^{\prime}+r^{\prime}, j}$, for $j^{\prime}=1, \ldots, r^{\prime}$.

(2) By the classification of causal compactifications the only $(\mathfrak{g}, \mathfrak{h}, \tau)$ with nontrivial center are those described by [B97, Corollary 5.3].

\section{Hardy spaces.}

We will from now on allways assume that $\mathfrak{g}$ is noncompact. Proposition 2.4(4) asserts that $\left(\mathfrak{g}_{s}, \mathfrak{h}_{s}, \tau \mid \mathfrak{g}_{s}\right)$ is a symmetric Lie algebra of Hermitian type in the sense of [ÓØ88, Ó91, HÓØ91] where we have a theory of Hardy spaces. In this section we generalize this to our reductive setting. Let $\mathfrak{t}$ be a Cartan algebra of $\mathfrak{g}$, with $\mathfrak{c}\left(\mathfrak{q}_{k}\right) \subset \mathfrak{a} \subset \mathfrak{t} \subset \mathfrak{k}$, and $\Delta\left(\mathfrak{g}_{\mathbb{C}}, \mathfrak{t}_{\mathbb{C}}\right)$ respectively $\Delta:=\Delta\left(\mathfrak{g}_{\mathbb{C}}, \mathfrak{a}_{\mathbb{C}}\right)$ the corresponding root systems. We may assume without loss of generality 
$\mathfrak{t} \subset \mathfrak{t}_{1}$. Indeed, if $\mathfrak{t}_{1}^{-} \cap \mathfrak{q}_{1 \sigma}=\{0\}$ by Proposition 2.4 then $\left[\mathfrak{t}, \mathfrak{t}_{1}^{-}\right]=\{0\}$ and (since the constructions in the last section needed only $\mathfrak{t}_{1}^{-}$) we can assume $\mathfrak{t}+\mathfrak{t}_{1}^{-} \subset \mathfrak{t}_{1}$. For the remaining (four) cases this is immediate from the realization of their causal compactifications ([B97]). We assume all introduced root systems to be lexicographically ordered with respect to the flag $\mathfrak{c}\left(\mathfrak{q}_{k}\right) \subset \mathfrak{a} \subset \mathfrak{t} \subset \mathfrak{t}+\mathfrak{t}_{1}^{-} \subset \mathfrak{t}_{1}$. Especially we have

$$
\mathfrak{p}^{ \pm}:=\bigoplus_{\alpha \in \Delta^{+}\left(\mathfrak{p}_{\mathbb{C}}, \mathfrak{t}_{\mathbb{C}}\right)} \mathfrak{g}_{\mathbb{C}, \pm \alpha} \subset \mathfrak{p}_{1}^{ \pm}
$$

Let $\Delta_{n}:=\Delta\left(\mathfrak{p}_{\mathbb{C}}, \mathfrak{a}_{\mathbb{C}}\right), \Delta_{n}^{+}:=\Delta\left(\mathfrak{p}^{+}, \mathfrak{a}_{\mathbb{C}}\right), \Delta_{k}:=\Delta\left(\mathfrak{k}_{\mathbb{C}}, \mathfrak{a}_{\mathbb{C}}\right)$, etc. Let $H_{\alpha} \in i \mathfrak{a}$ denote the co-root determined by the two conditions

$$
H_{\alpha} \in\left[\mathfrak{g}_{\mathbb{C}, \alpha}, \mathfrak{g}_{\mathbb{C},-\alpha}\right] \cap i \mathfrak{a} \quad \text { and } \quad \alpha\left(H_{\alpha}\right)=2 .
$$

Assume for the moment that $\left(\mathfrak{g}_{s}, \mathfrak{h}_{s}, \tau \mid \mathfrak{g}_{s}\right)$ is irreducible. Recall that for this case in $\mathfrak{q}$ we have the minimal closed cone $C_{\text {min }}$ and maximal closed cone $C_{\max }$ given by

$$
C_{\text {min }}=\overline{\operatorname{convAd}(H) Z_{2}}=C_{\min , s} \subset \mathfrak{g}_{s}
$$

and

$$
C_{\text {max }}=C_{\text {min }}^{*}=\mathfrak{z}+C_{\text {max }, s}
$$

where the subscribt ${ }_{s}$ indicates the corresponding cone in $\mathfrak{g}_{s}$. We have by [HÓ96, Chap. 4.2]

$$
c_{\min }:=C_{\min } \cap \mathfrak{a}=-i \overline{\sum_{\alpha \in \Delta_{n}^{+}} \mathbb{R}^{+} H_{\alpha}}
$$

and

$$
c_{\max }:=C_{\max } \cap \mathfrak{a}=\left\{H \in \mathfrak{a} \mid \forall \alpha \in \Delta_{n}^{+}:-i \alpha(H) \geq 0\right\} .
$$

Lemma 3.1. Denote by $\mathrm{pr}_{z}$ respectively $\mathrm{pr}_{s}$ the projection onto $\mathfrak{z}$ respectively $\mathfrak{g}_{s}$ corresponding to the decomposition $\mathfrak{g}=\mathfrak{z} \oplus \mathfrak{g}_{s}$. Then the following holds:

(1) $\operatorname{pr}_{z}(C)=\mathfrak{z}$.

(2) $C_{s}:=\operatorname{pr}_{s}(C)$ is a regular $H_{s}$-invariant cone in $\mathfrak{q}_{s}$. In particular $C_{\min , s} \subset \operatorname{pr}_{s}(C) \subset C_{\max , s}$.

Proof. (1) If $\mathfrak{z} \neq\{0\}$ the center is spanned by $Z_{1}^{\prime}=\sum \epsilon_{j} i H_{j}$, with $\epsilon_{1}=$ $\ldots=\epsilon_{p}=1=-\epsilon_{p+1}=\ldots=-\epsilon_{r}$ ([B97, Prop. 5.5]). In this case we also have $\lambda_{j^{\prime}, j}=\delta_{j^{\prime}, j}$. We get from Proposition 2.4(6) for elements in $\mathfrak{a} \cap C^{o}$

$$
\sum a_{j} i H_{j}=a Z_{1}^{\prime}+X_{s}
$$

where $a_{j}<0$ and $X_{s} \in \mathfrak{g}_{s}$. Since both sums are orthogonal it follows $a=\frac{1}{r} \sum \epsilon_{j} a_{j}$, which can take any value.

(2) Obviously $C_{s}$ is a convex generating $H_{s}$-invariant cone in $\mathfrak{q}_{s}$ because $C$ is a convex generating $H_{s}$-invariant cone in $\mathfrak{q}$. Assume that $C_{s}$ is not 
pointed. Let $\mathfrak{q}_{2}:=C_{s} \cap-C_{s}$. Then $\mathfrak{g}_{2}:=\left[\mathfrak{q}_{2}, \mathfrak{q}_{2}\right] \oplus \mathfrak{q}_{2}$ is a subalgebra of $\mathfrak{g}_{s}$. As $C$ is $\theta$-stable it follows that $\mathfrak{g}_{2}$ is $\theta$-stable and hence reductive. We have $\left[\mathfrak{h}_{s}, \mathfrak{g}_{2}\right] \subset \mathfrak{g}_{2}$ because $C$ is $H_{s}$-stable and because of the Jacobi identity. Let $\mathfrak{q}_{2}^{\perp}$ be the orthogonal complement to $\mathfrak{q}_{2}$ (with respect to the inner product $(X, Y)=-B(X, \theta(Y)))$. Let $Z \in \mathfrak{q}_{2}$ and $Y \in \mathfrak{q}_{2}^{\perp}$. Then

$$
-B([Z, Y], \theta([Z, Y]))=-B(Y,[Z,[\theta(Z), \theta(Y)]])=0
$$

because $[\theta(Z), \theta(Y)] \in \mathfrak{h}_{s}$. It follows that $\mathfrak{g}_{2}$ is a $\tau$-stable ideal in $\mathfrak{g}_{s}$. But $\left(\mathfrak{g}_{s}, \mathfrak{h}_{s}, \tau \mid \mathfrak{g}_{s}\right)$ is by [B97, Prop. 5.5] irreducible, hence $\mathfrak{q}_{2}=\mathfrak{q}_{s}$. It follows in particular that $\pm H_{\alpha} \in C_{s}$ for all $\alpha \in \Delta_{n}$. Choose a Lie algebra homomorphism

$$
\varphi_{\alpha}: \mathfrak{s u}(1,1) \rightarrow \mathfrak{g}_{s}
$$

such that

$$
\varphi_{\alpha}\left(\left(\begin{array}{cc}
i & 0 \\
0 & -i
\end{array}\right)\right)=i H_{\alpha}
$$

and

$$
T_{\alpha}:=\varphi_{\alpha}\left(\left(\begin{array}{ll}
0 & 1 \\
1 & 0
\end{array}\right)\right) \in \mathfrak{h}_{s}
$$

([ÓØ88, p. 134]). Let $h_{t}:=\exp t T_{\alpha}$ then a simple $S U(1,1)$-calculation shows that

$$
\operatorname{Ad}\left(h_{t}\right) H_{\alpha}+\operatorname{Ad}\left(h_{-t}\right) H_{\alpha}=2 \cosh (2 t) H_{\alpha} .
$$

Choose $Z \in \mathfrak{z}$ such that $Z+H_{\alpha} \in C$. Then

$$
\lim _{t \rightarrow \infty}\left(\frac{1}{\cosh (2 t)}\left(\operatorname{Ad}\left(h_{t}\right)\left(Z+H_{\alpha}\right)+\operatorname{Ad}\left(h_{-t}\right)\left(Z+H_{\alpha}\right)\right)\right)=2 H_{\alpha} \in C .
$$

Similarily one shows that $-2 H_{\alpha} \in C$. This contradicts the fact, that $C$ is pointed. Hence $C_{s}$ is also pointed.

The cone $C_{k}$ is minimal in $\mathfrak{q}_{1 \eta}$ and is generated by $\operatorname{Ad}\left(G_{1}^{\eta}\right) Z^{0}$. A minimal extension of $C_{k}$ to a $G_{1}$-invariant cone in $\mathfrak{g}_{1}$ is $W_{k}$, the minimal cone in $\mathfrak{g}_{1}$ generated by $\operatorname{Ad}\left(G_{1}\right) Z^{0}$ ([HÓ96, Chap. 4.2]). As $Z^{0} \in \mathfrak{q} \cap \mathfrak{k}$ it follows that $W_{k}$ is $\sigma$-invariant. For a subset $D \subset W_{k}$ we define

$$
\Gamma_{1}(D):=G_{1} \exp i D \subset G_{1 \mathbb{C}} .
$$

It is known that the Ol'shanskir semigroup $\Gamma_{1}\left(W_{k}\right)$ is a closed semigroup in $G_{1 \mathbb{C}}$ and that

$$
\Gamma_{1}\left(W_{k}\right)^{o}=\Gamma_{1}\left(W_{k}^{o}\right) \simeq G_{1} \times i W_{k}^{o},
$$

where the diffeomorphism is given by $(g, i X) \mapsto g \exp i X$.

Theorem 3.2. For $W:=W_{k} \cap \mathfrak{g}$ the following holds:

(1) $W=W_{k}^{\sigma}=\operatorname{pr}_{\mathfrak{g}}\left(W_{k}\right)$, where $\operatorname{pr}_{\mathfrak{g}}: \mathfrak{g}_{1} \rightarrow \mathfrak{g}$ denotes the orthogonal projection. 
(2) $W$ is a regular $G$-invariant cone in $\mathfrak{g}$ such that $W \cap \mathfrak{q}=\operatorname{pr}_{\mathfrak{q}}(W)=C$, where $\operatorname{pr}_{\mathfrak{q}}: \mathfrak{g} \rightarrow \mathfrak{q}$ denotes the orthogonal projection, i.e., $W$ is a $G$-invariant extension of $C$.

(3) $W^{o}=\operatorname{Ad}(G)\left(\mathfrak{t} \cap W^{o}\right)$.

(4) Let $\Gamma(W):=G \exp i W$. Then

$$
\Gamma(W)=\Gamma_{1}\left(W_{k}\right)^{\sigma}=\Gamma_{1}\left(W_{k}\right) \cap G_{1 \mathbb{C}}^{\sigma} .
$$

Thus $\Gamma(W)$ is a closed semigroup in $G_{\mathbb{C}}:=G_{1, \mathbb{C}}^{\sigma}$.

(5) $\Gamma(W)^{o}=G \exp i W^{o}=: \Gamma\left(W^{o}\right)$, and

$$
G \times i W^{o} \rightarrow \Gamma\left(W^{o}\right) \quad(g, i X) \mapsto g \exp i X
$$

is a diffeomorphism.

Proof. (1), (2), and (3) follow in the same way as the corresponding claims for the cones $C_{k}$ and $C$, using also the fact that $W_{k}$ is an extension of $C_{k}$.

(4) Obviously $\Gamma(W) \subset \Gamma_{1}\left(W_{k}\right)^{\sigma}$, and $\Gamma(W) \subset \Gamma_{1}\left(W_{k}\right) \cap G_{\mathbb{C}}$. Let $\gamma=$ $g \exp i X \in \Gamma_{1}\left(W_{k}\right)^{\sigma}, g \in G_{1}$ and $X \in W_{k}$. Then

$$
\sigma(g) \exp i \sigma(X)=g \exp i X \text {. }
$$

Because of the uniqueness of the decomposition in $\Gamma_{1}\left(W_{k}\right)$ we get $\sigma(g)=g$ and $\sigma(X)=X$. Hence $\Gamma(W)=\Gamma(W)^{\sigma}$. Assume now that $\gamma=g \exp i X \in$ $G_{\mathbb{C}} \cap \Gamma_{1}\left(W_{k}\right), g \in G_{1}, X \in W_{k}$. Then the same argument shows that $\sigma(g)=g$ and $\sigma(X)=X$. Hence $g \in G_{1}^{\sigma}$ and $X \in W_{k}^{\sigma}=W$. Hence $\gamma \in \Gamma(W)$. It follows that $\Gamma(W)$ is a closed semigroup.

(5) These claims follow by (4) and the corresponding facts for $\Gamma_{1}\left(W_{k}\right)$.

With these results it is straightforward to generalize the constructions of the holomorphic discrete series for $G / H$ ([0́Ø88, ÓØ91]) and the Hardy spaces ([HÓØ91]) to our setting.

To be complete we collect here the results needed by us. A survey is also given in [HÓ96, Chap. 7]. Let

$$
\rho:=\frac{1}{2} \sum_{\alpha \in \Delta^{+}} \operatorname{dim} \mathfrak{g}_{\mathbb{C}, \alpha} \alpha .
$$

We have that $\mathfrak{p}^{+}$and $\mathfrak{p}^{-}$are abelian subalgebras, $\mathfrak{g}_{\mathbb{C}}=\mathfrak{p}^{+} \oplus \mathfrak{k}_{\mathbb{C}} \oplus \mathfrak{p}^{-}$, and $\left[\mathfrak{k}_{\mathbb{C}}, \mathfrak{p}^{ \pm}\right] \subset \mathfrak{p}^{ \pm}$. With $P^{ \pm}:=\exp \left(\mathfrak{p}^{ \pm}\right)$we have $G \subset P^{+} K_{\mathbb{C}} P^{-} \cap H_{\mathbb{C}} K_{\mathbb{C}} P^{+}$, and for $x \in P^{+} K_{\mathbb{C}} P^{-}$respectively $H_{\mathbb{C}} K_{\mathbb{C}} P^{+}$we define $p^{ \pm}(x) \in P^{ \pm}, k_{\mathbb{C}}(x) \in K_{\mathbb{C}}$, and $k_{H}(x) \in K_{\mathbb{C}}$ (only the class in $K_{\mathbb{C}} \cap H_{\mathbb{C}} \backslash K_{\mathbb{C}}$ is well-defined) by

$$
x=p^{+}(x) k_{\mathbb{C}}(x) p^{-}(x)
$$

respectively

$$
x \in H_{\mathbb{C}} k_{H}(x) P^{+} .
$$


Let $\left(\delta, V_{\delta}\right)$ be a holomorphic irreducible representation of $K_{\mathbb{C}}$ with nonzero $K_{\mathbb{C}}^{\tau}$-fixed vector $\nu$ and $\delta \mid K$ unitary. For $\check{\delta}$ the contragredient representation,

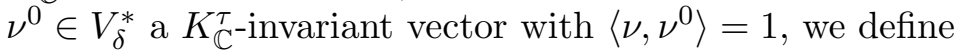

$$
\Phi_{\delta}: P^{+} K_{\mathbb{C}} H_{\mathbb{C}} / H_{\mathbb{C}} \rightarrow \mathbb{C}, g H \mapsto\left\langle\nu, \check{\delta}\left(k_{H}\left(g^{-1}\right)^{-1}\right) \nu^{0}\right\rangle .
$$

This is a well-defined holomorphic function because $\nu^{0}$ is $K_{\mathbb{C}} \cap H_{\mathbb{C}}$-invariant. We denote the restriction of $\Phi_{\delta}$ to $G / H$ simply by $\Phi_{\delta}$. With this convention notice that $\Phi_{\delta} \mid\left(G_{s} / H_{s}\right)=\Phi_{\delta_{s}}$ with $\delta_{s}=\delta \mid\left(K_{s, \mathbb{C}}\right)$. Let $Z_{\mathbb{C}}$ be the complex torus given by $\exp \mathfrak{z} \mathbb{C}$. Define $\chi_{\delta}: Z_{\mathbb{C}} \rightarrow \mathbb{C}$ by

$$
\delta(z)=\chi_{\delta}(z) \mathrm{id} .
$$

Theorem 3.3 ([0́Ø91]). Let $\mu$ be the highest weight of $\delta$. Then the following holds:

(1) The function $\Phi_{\delta}$ is in $L^{2}(G / H)$ if and only if $\langle\mu+\rho, \alpha\rangle<0$ for all $\alpha \in \Delta_{n}^{+}$.

(2) Suppose that $\langle\mu+\rho, \alpha\rangle<0$ for all $\alpha \in \Delta_{n}^{+}$. Let $H_{\delta} \subset L^{2}(G / H)$ be the $G$-module generated by $\Phi_{\delta}$. Then $H_{\delta}$ is an irreducible highest weight module.

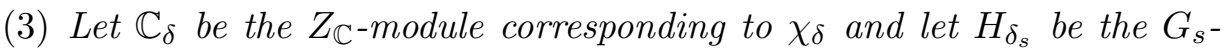
module generated by $\Phi_{\delta_{s}}$, then $H_{\delta} \simeq \mathbb{C}_{\delta} \otimes H_{\delta_{s}}$.

(4) Let $E \subset L^{2}(G / H)$ be an irreducible highest weight module. Then there exists a representation $\delta$ such that $E \simeq H_{\delta}$ and the multiplicity of $E$ in $L^{2}(G / H)$ is one.

Proof. (1) For $g \in G$ choose $z \in Z$ and $g_{s} \in G_{s}$ such that $g=z g_{s}$. Then $\Phi_{\delta}(g H)=\chi_{\delta}(z) \Phi_{\delta_{s}}\left(g_{s} H_{s}\right)$, in particular

$$
\left|\Phi_{\delta}(g H)\right|=\left|\Phi_{\delta_{s}}\left(g_{s} H\right)\right| .
$$

Hence $\Phi_{\delta} \in L^{2}(G / H)$ if $\langle\mu+\rho, \alpha\rangle<0$ for all $\alpha \in \Delta_{n}^{+}$by [ÓØ91, Theorem 5.2] and [HÓØ91, Theorem 3.3].

(2), (3) Denote the $G_{s}$-module generated by $\Phi_{\delta_{s}}$ in $L^{2}\left(G_{s} / H_{s}\right)$ by $H_{\delta_{s}}$. Then

$$
H_{\delta} \simeq \mathbb{C}_{\chi_{\delta}} \otimes H_{\delta_{s}}
$$

as a $Z \times G_{s}$ module. The module on the right hand side is an irreducible highest weight module by [ÓØ91, Theorem 5.2]. Hence $H_{\delta}$ must be irreducible.

(4) Assume that $E \subset L^{2}(G / H)$ is an irreducible highest weight module. Then $E \simeq \mathbb{C}_{\chi} \otimes E_{s}$, where $E_{s}$ is the restriction of $E$ to $G_{s}$ and $\mathbb{C}_{\chi}$ is the central character of $E$. The module $E_{s}$ is an irreducible highest weight module for $G_{s}$, and $E_{s} \subset L^{2}\left(G_{s} / H_{s}\right)$. By [HÓØ91, Theorem 3.3] it follows that

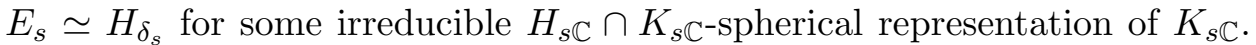
Let $\delta=\chi \otimes \delta_{s}$. Then $\delta$ is an irreducible $K_{\mathbb{C}} \cap H_{\mathbb{C} \text {-spherical representation }}$ of $K_{\mathbb{C}}$ and $E \simeq H_{\delta}$. The statement about the multiplicity follows now from [ÓØ91, Theorem 7.2]. 
These representations $\epsilon_{\delta}$ on $H_{\delta}$ form the holomorphic discrete series of $G / H$. Let $\pi$ be a unitary representation of $G$ an the Hilbert space $V$. Denote the space of smooth vectors by $V^{\infty}$. Denote by $\pi^{\infty}$ the derived representation of $\mathfrak{g}$ on $V^{\infty}$. Define the cone of negative elements by

$$
C(\pi):=\left\{X \in \mathfrak{g} \mid \forall u \in V^{\infty}:\left(i \pi^{\infty}(X) u \mid u\right) \leq 0\right\} .
$$

Then $C(\pi)$ is a $G$-invariant cone in $\mathfrak{g}$. The representation $\pi$ is called $W$ admissible if $C(\pi) \supset W$. If $\pi$ is $W$-admissible then $\pi$ extends to a holomorphic representation of $\Gamma(W)$. (In fact, this is true for $W$ replaced by an arbitrary invariant cone.)

Proposition 3.4. For $W=W_{k} \cap \mathfrak{g}$ the representation $\left(\epsilon_{\delta}, H_{\delta}\right)$ is $W$-admissible if and only if

$$
i \mu\left(\mathfrak{a} \cap C^{o}\right)=\left(i d \chi_{\delta}+i \mu_{s}\right)\left(\mathfrak{a} \cap C^{o}\right) \leq 0 .
$$

Proof. Since $W^{o}=\operatorname{Ad}(G)\left(\mathfrak{t} \cap W^{o}\right)$ and $\epsilon_{\delta}$ is unitary, $W$-admissibility is equivalent to

$$
\left(i \epsilon_{\delta}^{\infty}\left(\mathfrak{t} \cap W^{o}\right) u \mid u\right) \leq 0
$$

for all $u \in H_{\delta}^{\infty}$. Let $W(\mathfrak{k}, \mathfrak{t})$ be the Weyl group of $\Delta(\mathfrak{k}, \mathfrak{t})$. As $H_{\delta}$ is an irreducible highest weight module we have by Poincaré-Birkhoff-Witt $\mu_{\lambda}=\mu_{+}-\sum_{\alpha \in \Delta^{+}\left(\mathfrak{p}_{\mathbb{C}}, \mathfrak{t}_{\mathbb{C}}\right)} n_{\alpha} \alpha$, with $n_{\alpha} \geq 0$ and $\mu_{+} \in \operatorname{conv}(W(\mathfrak{k}, \mathfrak{t}) \mu)$, for all occuring weights $\mu_{\lambda}$. Since $\mathfrak{t} \cap W \subset \mathfrak{t}_{1} \cap W_{k}=-\sum_{\alpha \in \Delta^{+}\left(\mathfrak{p}_{1 \mathbb{C}}, \mathfrak{t}_{1 \mathbb{C}}\right)} \mathbb{R}_{0}^{+} i H_{\alpha}$ (the $H_{\alpha} \in \mathfrak{t}_{1 \mathbb{C}}$ to $\alpha \in \Delta\left(\mathfrak{g}_{1 \mathbb{C}}, \mathfrak{t}_{1 \mathbb{C}}\right)$ defined as the $H_{\alpha}$ to $\alpha \in \Delta$ ) ([HÓ96, p. 102]) such that $i \alpha(\mathfrak{t} \cap W) \geq 0$ for all noncompact roots $\alpha \in \Delta^{+}\left(\mathfrak{p}_{\mathbb{C}}, \mathfrak{t}_{\mathbb{C}}\right)$, the condition $(*)$ remains to be checked for the vectors in $\operatorname{conv}(W(\mathfrak{k}, \mathfrak{t}) \mu)$. Now, since $W$ is $G$-invariant, $\mathfrak{t} \cap W^{o}$ is $W(\mathfrak{k}, \mathfrak{t})$-invariant, and we have to check (*)

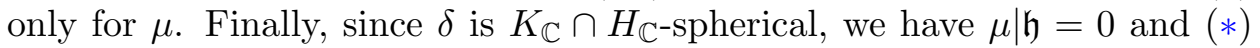
reduces to the claimed condition.

Remark. For $\mathfrak{g}$ semisimple and $\mathfrak{a} \cap C=c_{\text {min }}$ it is known by [HÓØ91] that all $\left(\epsilon_{\delta}, V_{\delta}\right)$ are admissible.

Let $\Xi(C):=\Gamma(-W) x_{o} \subset G_{\mathbb{C}} / H_{\mathbb{C}}$, where $x_{o}=e H_{\mathbb{C}}$. As in [HÓØ91, Lemma 1.3] we have $\Xi(C) \simeq G \times_{H}(-i C)$ and for $\Xi\left(C^{o}\right):=\Gamma\left(-W^{o}\right) x_{o}$ we have $\Xi(C)^{o}=\Xi\left(C^{o}\right)$. Thus $\Xi\left(C^{o}\right)$ is an open complex submanifold of $G_{\mathbb{C}} / H_{\mathbb{C}}$. Notice that $\Gamma(-W)=\Gamma(W)^{-1}$. As $\Gamma(W) \Gamma\left(W^{o}\right) \subset \Gamma\left(W^{o}\right)$ it follows that $\Gamma\left(-W^{o}\right) \Xi(C) \subset \Xi\left(C^{o}\right)$. Thus $\Gamma(W)$ acts on functions defined on $\Xi(C)$ respectively $\Xi\left(C^{o}\right)$ by

$$
(\gamma \cdot f)(x)=f\left(\gamma^{-1} x\right)
$$

and $\left.(\gamma \cdot f)\right|_{G / H}$ is well-defined for $\gamma \in \Gamma\left(W^{o}\right)$. Define the Hardy norm on holomorphic functions on $\Xi\left(C^{o}\right)$ by

$$
\|f\|_{H}:=\sup _{\gamma \in \Gamma^{0}}\|\gamma \cdot f\|_{L^{2}(G / H)} .
$$


Definition. The Hardy space $H_{2}(C)$ on $G / H$ is

$$
H_{2}(C):=\left\{f: \Xi\left(C^{o}\right) \rightarrow \mathbb{C} \mid f \text { is holomorphic and }\|f\|_{H}<\infty\right\} .
$$

Denote by $T$ the representation of $\Gamma(W)$ in $H_{2}(C)$ induced by the leftregular action and by $\lambda$ the left-regular representation of $G$ in $L^{2}(G / H)$. The proof of the following theorem then generalizes without any change (or simply using again that $G=Z G_{s}$ ).

Theorem 3.5 ([HÓØ91]). (1) The Hardy space $H_{2}(C)$ is a Hilbert space with norm $\|\cdot\|_{H}$.

(2) There is an isometry $\beta: H_{2}(C) \rightarrow L^{2}(G / H)$ given by

$$
\beta(f)=\lim _{\Gamma\left(W^{o}\right) \ni \gamma \rightarrow 1} \gamma \cdot f,
$$

where the limit is in $L^{2}(G / H)$.

(3) The boundary value map $\beta$ is an intertwining operator for the $G$ actions, i.e., $\beta T(g)=\lambda(g) \beta$ for $g \in G$.

(4) The representation $T$ is a holomorphic representation of $\Gamma(W)$ in $\mathrm{H}_{2}(\mathrm{C})$.

(5) The image of $\beta$ in $L^{2}(G / H)$ is the direct sum of all the holomorphic discrete series that are $W$-admissible and each occurs with multiplicity one.

(6) Assume that $w \in \Xi\left(C^{o}\right)$. Then the evaluation map $H_{2}(C) \ni f \mapsto$ $f(w) \in \mathbb{C}$ is continuous. Let $K_{w} \in H_{2}(C)$ be such that $f(w)=(f)$ $\left.K_{w}\right)$, where $(\cdot \mid \cdot)$ denotes the scalar product on $H_{2}(C)$. Then the map $(z, w) \mapsto K(z, w):=K_{w}(z)$ is holomorphic in the first variable and antiholomorphic in the second variable. We have:

a) $\overline{K(w, z)}=K(z, w)$.

b) Let $\gamma \in \Gamma(-W)$, and let $z, w \in \Xi\left(C^{o}\right)$. Then

$$
K(\gamma \cdot z, w)=K\left(z, \gamma^{*} \cdot w\right),
$$

with $(g \exp i X)^{*}:=\exp (i X) g^{-1}$.

c) There exists a holomorphic $H$-invariant function $\Theta_{K}: \Xi\left(C^{o}\right) \rightarrow \mathbb{C}$ such that

$$
K\left(\gamma_{1} \cdot x_{o}, \gamma_{2} \cdot x_{o}\right)=\Theta_{K}\left(\gamma_{2}^{*} \gamma_{1} \cdot x_{o}\right) .
$$

(7) Suppose that $z=\gamma \cdot x_{o} \in \Xi\left(C^{o}\right)$. Then $w \mapsto K(z, w)$ extends to a smooth map on $\Xi(C)$, and the inverse of $\beta$ is given by

$$
F(z)=\int_{G / H} f(x) K(z, x) d x=\int_{G / H} f(\dot{g}) \Theta_{K}\left(g^{-1} \gamma \cdot x_{o}\right) d \dot{g},
$$

where $\dot{g}=g \cdot x_{o}$, and $d \dot{g}$ is the $G$-invariant measure on $G / H$ normalized by $d g=d h d \dot{g}$.

We note for later use that the extension of $\Phi_{\delta}$ from $G / H$ to $\Xi\left(C^{o}\right)$ is given by the defining formula, as $\Gamma(-W) \subset H_{\mathbb{C}} K_{\mathbb{C}} P^{+}$([HÓØ91, Lemma 3.6]). 
Example (continued). Since $\mathfrak{g}=\mathfrak{k} \cap \mathfrak{q}=\mathfrak{a}$ we have by Proposition 2.4

$$
C^{o}=\mathbb{R}^{+} Z^{0}=\mathbb{R}^{+}\left(\begin{array}{cc}
-i & 0 \\
0 & i
\end{array}\right)=W^{o}
$$

and further

$$
\Gamma(-W)=\left\{\left(\begin{array}{cc}
a & 0 \\
0 & a^{-1}
\end{array}\right)|| a \mid \leq 1\right\} .
$$

The extension of $\Phi: G / H \rightarrow \check{S}_{1}$ to a $G_{\mathbb{C} \text {-invariant holomorphic map is }}$

$$
\Phi: G_{\mathbb{C}} / H_{\mathbb{C}} \rightarrow \mathbb{C} \quad\left(\begin{array}{cc}
a & 0 \\
0 & a^{-1}
\end{array}\right) H_{\mathbb{C}} \mapsto-a^{2},
$$

such that

$$
\Phi\left(\Xi(C)^{o}\right)=\Phi\left(\Gamma\left(-W^{o}\right) x_{o}\right)=\{z \in \mathbb{C}|0<| z \mid<1\} .
$$

We describe the Hardy space using this explicit realization of $\Xi\left(C^{o}\right)$. Since $G$ operates by rotations, the Hardy norm is given by

$$
\|f\|_{H}=\sup _{0<|z|<1} \frac{1}{\pi} \int_{0}^{\pi}\left|f\left(e^{2 i \varphi} z\right)\right|^{2} d \varphi
$$

and, using the Laurent series representation of $f$, we get

$$
\begin{aligned}
H_{2}(C)=\{f:\{z \in \mathbb{C}|0<| z \mid<1\} \rightarrow \mathbb{C} \mid f(z)= & \sum_{n=0}^{\infty} a_{n} z^{n} \\
& \text { with } \left.\sum_{n=0}^{\infty}\left|a_{n}\right|^{2}<\infty\right\} .
\end{aligned}
$$

We can describe this space also as sum of $W$-admissible representations. Identifying $G / H$ via $\Phi$ with $S^{1}$, the holomorphic discrete series representations are the characters

$$
\Phi_{n}: S^{1} \rightarrow \mathbb{C}, z \mapsto z^{n}, n \in \mathbb{Z} .
$$

All these functions have obviously a holomorphic continuation to $\Xi\left(C^{o}\right)$. Checking which of these representations are $W$-admissible, we get the condition $n \geq 0$, proving Theorem 3.5(5) for this case.

\section{Characterization of $\Phi(G / H)$.}

The Weyl group for the root system $\Delta\left(\mathfrak{k}_{1 \mathbb{C}}, \mathfrak{t}_{1 \mathbb{C}}\right)$ of compact roots acts by permutations on the positive noncompact roots $\Delta^{+}\left(\mathfrak{p}_{1 \mathbb{C}}, \mathfrak{t}_{1 \mathbb{C}}\right)$. Therefore

$$
\rho_{1, n}:=\frac{1}{2} \sum_{\alpha \in \Delta^{+}\left(\mathfrak{p}_{1 \mathbb{C}}, \mathfrak{t}_{1 \mathbb{C}}\right)} \operatorname{dim}_{\mathbb{C}} \mathfrak{g}_{1 \mathbb{C}, \alpha} \alpha \in i \mathfrak{t}_{1}^{*}
$$

is zero on the orthogonal complement of $\mathfrak{z}\left(\mathfrak{k}_{1}\right)$ in $\mathfrak{t}_{1}$. We define $\Delta_{1}:=$ $\Delta\left(\mathfrak{g}_{1 \mathbb{C}}, \mathfrak{t}_{1 \mathbb{C}}^{-}\right)$and the positive roots $\Delta_{1}^{+}$by restricting the roots $\Delta^{+}\left(\mathfrak{g}_{1 \mathbb{C}}, \mathfrak{t}_{1 \mathbb{C}}\right)$ 
to $\mathfrak{t}_{1 \mathbb{C}}^{-}$. Since $\left(\mathfrak{g}_{1}, \mathfrak{k}_{1}, \theta\right)$ is assumed to be Hermitian symmetric of tube type, and, by abuse of notation, identifying the strongly orthogonal roots $\gamma_{i}$ with elements of $\left(\mathfrak{t}_{1 \mathbb{C}}^{-}\right)^{*}$, by the Theorem of Moore $([\mathbf{H 7 8}$, p. 528] or $[\mathbf{H 9 4}$, p. 460])

$$
\Delta_{1}=\left\{ \pm \frac{1}{2}\left(\gamma_{i}+\gamma_{j}\right) \mid 1 \leq i \leq j \leq r\right\} \cup\left\{ \pm \frac{1}{2}\left(\gamma_{i}-\gamma_{j}\right) \mid 1 \leq i<j \leq r\right\} .
$$

Thereby the positive noncompact roots are

$$
\Delta_{1, n}^{+}=\left\{\frac{1}{2}\left(\gamma_{i}+\gamma_{j}\right) \mid 1 \leq i \leq j \leq r\right\},
$$

the root spaces $\mathfrak{g}_{1 \mathbb{C}, \gamma_{i}}$ are one dimensional, and root spaces correponding to roots $\frac{1}{2}\left(\gamma_{i} \pm \gamma_{j}\right), i \neq j$, have all the same dimension $d$. As the restriction of a noncompact root to $\mathfrak{t}_{1 \mathbb{C}}^{-}$is allways nonzero, we get immediately

$$
\rho_{1, n}=\frac{1}{2}\left(1+\frac{d(r-1)}{2}\right)\left(\gamma_{1}+\ldots+\gamma_{r}\right) .
$$

For the moment we prefer to work with another Cartan algebra then $\mathfrak{t}_{1}$. Therefore, let $\mathfrak{t}_{1}^{+}$be the orthogonal complement of $\mathfrak{t}_{1}^{-}$in $\mathfrak{t}_{1}$, then the Cayley transform is the identity on $\mathfrak{t}_{1 \mathbb{C}}^{+}$and maps $\mathfrak{t}_{1 \mathbb{C}}^{-}$onto $\mathfrak{a}_{p \mathbb{C}}$. For $\alpha \in \Delta\left(\mathfrak{g}_{1 \mathbb{C}}, \mathfrak{t}_{1 \mathbb{C}}\right)$ we define the Cayley transformed root $\alpha^{c}$ by

$$
\alpha^{c}:=\alpha \circ \operatorname{Ad}\left(c^{-1}\right) \in \Delta\left(\mathfrak{g}_{1 \mathbb{C}}, \mathfrak{t}_{1 \mathbb{C}}^{+} \oplus \mathfrak{a}_{p \mathbb{C}}\right)=: \Delta_{1}^{c},
$$

and the positive system $\Delta_{1}^{c,+}$ by the transformed roots of $\Delta^{+}\left(\mathfrak{g}_{1 \mathbb{C}}, \mathfrak{t}_{1 \mathbb{C}}\right)$.

We define

$$
\rho_{+}:=\operatorname{Ad}\left(c^{-1}\right)^{*} \rho_{1, n}=\frac{1}{2}\left(1+\frac{d(r-1)}{2}\right)\left(\gamma_{1}^{c}+\cdots+\gamma_{r}^{c}\right) \in\left(\mathfrak{t}_{1 \mathbb{C}}^{+}+\mathfrak{a}_{p \mathbb{C}}\right)^{*},
$$

which is zero on the orthogonal complement of $\mathbb{C} X^{0}$. We denote by $\left(\pi_{m}, V_{m}\right)$ the irreducible finite-dimensional representation of $G_{1 \mathbb{C}}$ with lowest weight $-m \rho_{+}$, if it exists.

We will need the Riemannian dual algebra $\mathfrak{g}_{1}^{d}$ associated to $\left(\mathfrak{g}_{1}, \mathfrak{g}, \sigma\right)$ for the proof of the next proposition

$$
\mathfrak{g}_{1}^{d}:=\mathfrak{k}_{1} \cap \mathfrak{g}+i\left(\mathfrak{p}_{1} \cap \mathfrak{g}\right)+\mathfrak{p}_{1} \cap \mathfrak{q}_{1 \sigma}+i\left(\mathfrak{k}_{1} \cap \mathfrak{q}_{1 \sigma}\right) .
$$

The involution $\sigma$ restricted to $\mathfrak{g}_{1}^{d}$ is a Cartan involution. Hence $\mathfrak{k}_{1}^{d}:=\mathfrak{k}_{1} \cap$ $\mathfrak{g}+i\left(\mathfrak{p}_{1} \cap \mathfrak{g}\right)$ is a maximal compactly embedded subalgebra of $\mathfrak{g}_{1}^{d}$ and the corresponding orthogonal complement is

$$
\mathfrak{p}_{1}^{d}:=\mathfrak{p}_{1} \cap \mathfrak{q}_{1 \sigma}+i\left(\mathfrak{k}_{1} \cap \mathfrak{q}_{1 \sigma}\right) .
$$

Let $\hat{\mathfrak{a}} \subset \mathfrak{k}_{1} \cap \mathfrak{q}_{1 \sigma} \cap \mathfrak{z}_{\mathfrak{g}_{1}}(\mathfrak{b})$ be maximal abelian, then $\mathfrak{a}^{d}:=i \hat{\mathfrak{a}}+\mathfrak{b}$ is maximal abelian in $\mathfrak{p}_{1}^{d}$. For the root system $\Delta_{1}^{d}:=\Delta\left(\mathfrak{g}_{1}^{d}, \mathfrak{a}^{d}\right)$ we choose the positive roots $\Delta_{1}^{d,+}:=\Delta^{+}\left(\mathfrak{g}_{1}^{d}, \mathfrak{a}^{d}\right)$ compatible with $\Delta^{+}\left(\mathfrak{g}_{1}^{d}, \mathfrak{b}\right)=\Delta^{+}\left(\mathfrak{g}_{1}, \mathfrak{b}\right)=$ $\left\{\left.\alpha\right|_{\mathfrak{b}}\left|\alpha \in \Delta_{1}^{c,+}, \alpha\right|_{\mathfrak{b}} \neq 0\right\}$. 
Proposition 4.1. Let the notation be as above, then the following holds:

(1) There exists an irreducible finite-dimensional representation $\left(\pi_{2}, V_{2}\right)$ of $G_{1 \mathbb{C}}$ with lowest weight $-2 \rho_{+}$.

(2) Assume that $\mathfrak{g}_{1} \neq \mathfrak{s} p(2 n, \mathbb{R}), \mathfrak{s} o(2,2 k+1), n, k \geq 1$. Then there exists an irreducible finite-dimensional representation $\left(\pi_{1}, V_{1}\right)$ of $G_{1 \mathbb{C}}$ with lowest weight $-\rho_{+}$.

(3) The weight space $V_{m,-m \rho_{+}}$is left pointwise fixed by the identity component of $M_{\mathbb{C}}^{\prime}$, where $M_{\mathbb{C}}^{\prime}$ is defined by the Langlands decomposition $P^{\prime} \simeq M^{\prime} \times \exp \left(\mathbb{R} X^{0}\right) \times Q_{1 \eta}^{-}$. For $m=4$ it is fixed pointwise by $M_{\mathbb{C}}^{\prime}$ and, if $\mathfrak{g}_{1} \neq \mathfrak{s} p(2 n, \mathbb{R}), \mathfrak{s o}(2,2 k+1), n, k \geq 1$, the same is true for $m=2$.

(4) There is a $m \in\{1,2,4,8\}$ such that $\pi_{m}$ is $G_{\mathbb{C}}$-spherical. In this case, let $(\cdot \mid \cdot)$ be a scalar product, invariant under the analytic subgroup to $\mathfrak{k}_{1}+i \mathfrak{p}_{1}$, then we can choose $v_{m} \in V_{m,-m \rho_{+}}$and $\xi_{m} \in V_{m}^{G_{\mathbb{C}}}$ such that $\left(v_{m} \mid \xi_{m}\right)=1$.

Proof. (1), (2) The first two assertions are [ÓØ99, Theorem 2.6].

(3) Let $\widetilde{\mathfrak{a}}$ be the orthogonal complement of $\mathbb{R} X^{0}$ in $\mathfrak{a}_{p}$ and $\Delta_{0}:=\{\alpha \in$ $\left.\Delta_{1}^{c} \mid \alpha\left(X^{0}\right)=0\right\}$ then

$$
\mathfrak{m}_{\mathbb{C}}=\mathfrak{t}_{1 \mathbb{C}}^{+}+\widetilde{\mathfrak{a}}_{\mathbb{C}}+\sum_{\alpha \in \Delta_{0}} \mathfrak{g}_{1 \mathbb{C}, \alpha}
$$

Every lowest weight vector is invariant under $\mathfrak{t}_{1 \mathbb{C}}^{+}+\widetilde{\mathfrak{a}}$ and elements of root spaces $\mathfrak{g}_{1 \mathbb{C}, \alpha}$ with $-\alpha \in \Delta_{1}^{c,+}$. By the invariance of the weights under the Weyl group, for $\alpha \in \Delta_{1}^{c,+}$ with $\left\langle\alpha, \rho_{+}\right\rangle=0$ the weight space to $-m \rho_{+}+\alpha=$ $s_{\alpha}\left(-m \rho_{+}-\alpha\right)$ is trivial, hence $\mathfrak{m}_{\mathbb{C}}$ operates trivial on $V_{m,-m \rho_{+}}$. By the Theorem of Helgason $\pi_{m}\left(M_{\min }\right)$, with $M_{\min }:=Z_{K_{1}}\left(\mathfrak{a}_{p}\right)$, leaves the weight space pointwise fixed if and only if $\frac{\langle m \rho+, \alpha\rangle}{\langle\alpha, \alpha\rangle} \in \mathbb{Z}^{+}$for all $\alpha \in \Delta_{1}^{c,+}$. For $\alpha=\gamma_{j}^{c}$ this quotient is $\frac{m}{2}\left(1+\frac{d(r-1)}{2}\right)$, for $\alpha=\frac{1}{2}\left(\gamma_{i}^{c}+\gamma_{j}^{c}\right)$ it is $m\left(1+\frac{d(r-1)}{2}\right)$, and for $\alpha=\frac{1}{2}\left(\gamma_{i}^{c}-\gamma_{j}^{c}\right)$ it vanishes. This proofs the invariance under $M_{\min }$, for $m$ as in the assertion, by looking at the possible combinations for $d$ and $r$ $\left([\mathbf{H 7 8}\right.$, p. $530 \mathrm{ff}]$ or the table in [ÓØ99]). By $M_{\mathbb{C}}=M_{\min }\left\langle\exp \left(\mathfrak{m}_{\mathbb{C}}\right)\right\rangle$ the result now follows.

(4) By Helgason's Theorem $\pi_{m}$ is $G_{\mathbb{C}^{-} \text {-spherical if and only if }}$

$$
\frac{\left\langle m \rho_{+}, \alpha\right\rangle}{\langle\alpha, \alpha\rangle} \in \mathbb{Z}^{+}, \quad \forall \alpha \in \Delta_{1}^{d,+} .
$$

By definition $2 \rho_{1, n} \mid \mathfrak{b}$ is a linear combination of elements of $\Delta^{+}\left(\mathfrak{p}_{1 \mathbb{C}}, \mathfrak{b}\right)$ with positive integer coefficients. Therefore $2 \rho_{+}$is a linear combination with positive integer coefficients of elements of $\Delta^{+}\left(\mathfrak{g}_{1}, \mathfrak{b}\right)$. Now by [B88, p. 362] 
the system $\Delta\left(\mathfrak{g}_{1}, \mathfrak{b}\right)$ is a root system in the axiomatic sense such that

$$
2 \frac{\left\langle 2 \rho_{+}, \widetilde{\alpha}\right\rangle}{\langle\widetilde{\alpha}, \widetilde{\alpha}\rangle} \in \mathbb{Z} \quad \text { for } \quad \widetilde{\alpha} \in \Delta\left(\mathfrak{g}_{1}, \mathfrak{b}\right)
$$

For $\widetilde{\alpha}:=\left.\alpha\right|_{\mathfrak{b}}$, with $\alpha \in \Delta_{1}^{d}$, and $\widetilde{\alpha} \neq 0$ by [Ó87, Lemma 2.3] we have

$$
\langle\alpha, \alpha\rangle=n\langle\widetilde{\alpha}, \widetilde{\alpha}\rangle, \quad n \in\{1,2,4\},
$$

and $2 \alpha \in \Delta_{1}^{d}$ if $n=4$. Since a root $2 \alpha$ gives a more severe restriction then $\alpha$ we can assume $n \in\{1,2\}$, which implies the first assertion. The second assertion then is a consequence of the proof of Helgason's Theorem $[\mathbf{H 8 4}$, p. 534ff].

Example. We consider the causal compactification of $S O(2, n) / S O(1, n)$, as treated in [B97, Example 3.3]. Here $G_{1}=S O(2, n+1)$, i.e.,

$$
G_{1}=\left\{\left(\begin{array}{ll}
A & B \\
C & D
\end{array}\right) \in S L(n+3, \mathbb{R}) \mid \begin{array}{l}
{ }^{t} A A-{ }^{t} C C=I, \quad{ }^{t} A B={ }^{t} C D \\
{ }^{t} D D-{ }^{t} B B=I
\end{array}\right\},
$$

with Cartan involution $\theta(g)={ }^{t} g^{-1}, X^{0}=2\left(E_{1, n+3}+E_{n+3,1}\right)$, and

$$
\sigma=\operatorname{Ad}\left(\begin{array}{cc}
\operatorname{id}_{n+2} & 0 \\
0 & -1
\end{array}\right)
$$

Then $\mathfrak{q}_{1 \sigma}$ consists of matrices with nonzero entries only in the last row respectively column such that $\mathfrak{b}=\mathbb{R} X^{0}$ is maximal abelian in $\mathfrak{p}_{1}^{d}$, i.e., $\mathfrak{a}^{d}=\mathfrak{b}$. For the Riemannian dual algebra $\mathfrak{g}_{1}^{d}$ we get therefore, restricting the roots of $\Delta^{+}\left(\mathfrak{g}_{1}, \mathfrak{a}_{p}\right)=\left\{\gamma_{1}^{c}, \gamma_{2}^{c}, \frac{1}{2}\left(\gamma_{1}^{c} \pm \gamma_{2}^{c}\right)\right\}$ to $\mathfrak{b}$, the positive system $\Delta^{+}\left(\mathfrak{g}_{1}^{d}, \mathfrak{b}\right)=$ $\Delta^{+}\left(\mathfrak{g}_{1}, \mathfrak{b}\right)=\left\{\frac{1}{2}\left(\gamma_{1}^{c}+\gamma_{2}^{c}\right)\right\}$. By Helgason's Theorem, applied to $\left(\mathfrak{g}_{1}^{d}, \mathfrak{b}\right)$, the condition for $\pi_{m}$ to be $G$-spherical is now

$$
m\left(1+\frac{d(r-1)}{2}\right) \in \mathbb{Z}^{+}
$$

For our $\mathfrak{g}_{1}$ here $r=2$ and $d=n-1$ ([H78, p. 530ff] or the table in [ÓØ99]) such that $\pi_{2}$ is always $G$-spherical, whereas $\pi_{1}$ only for $n$ odd.

In a similar fashion one calculates the minimal $m$ 's for the other causal compactifications as given in the following table. Here $p$ and $q$ are nonnegative integers with $p+q=n$. In the case $(\mathfrak{s o} o(2, q) \oplus \mathfrak{s} o(p+1), \mathfrak{s} o(1, q) \oplus$ $\mathfrak{o}(p), \mathfrak{s} o(2, n+1))$ we also assume that $p \geq 2$ : 


\begin{tabular}{|l|l|}
\hline \multicolumn{1}{|c|}{$\left(\mathfrak{g}, \mathfrak{h}, \mathfrak{g}_{1}\right)$} & \multicolumn{1}{|c|}{ minimal $m$} \\
\hline$(\mathfrak{s}(\mathfrak{u}(p, q) \oplus \mathfrak{u}(p, q)), \mathfrak{s} u(p, q), \mathfrak{s} u(n, n))$ & 1 for $n$ even \\
$\left(\mathfrak{u}(2 p, 2 q), \mathfrak{s} p(p, q), \mathfrak{s} o^{*}(4 n)\right)$ & 2 for $n$ odd \\
$(\mathfrak{u}(p, q), \mathfrak{s} o(p, q), \mathfrak{s} p(n, \mathbb{R}))$ & 2 \\
& 1 for $n=3(\bmod 4)$ \\
& 2 for $n=1(\bmod 4)$ \\
$(\mathfrak{s} o(2, n-1) \oplus \mathfrak{s} o(2), \mathfrak{s} o(1, n-1)$, & 4 for $n$ even \\
$\mathfrak{s} o(2, n+1))$ & 1 for $n=3(\bmod 4)$ \\
& 2 for $n=1(\bmod 4)$ \\
$(\mathfrak{e} 6(-14) \oplus \mathfrak{s} o(2), \mathfrak{f} 4(-20), \mathfrak{e} 7(-25))$ & 4 for $n$ even \\
$\left(\mathfrak{s} o^{*}(2 n) \oplus \mathfrak{s} o^{*}(2 n), \mathfrak{s} o^{*}(2 n), \mathfrak{s} o^{*}(4 n)\right)$ & 2 \\
$(\mathfrak{s} p(n, \mathbb{R}) \oplus \mathfrak{s} p(n, \mathbb{R}), \mathfrak{s} p(n, \mathbb{R})$, & 4 \\
$\mathfrak{s} p(2 n, \mathbb{R}))$ & 1 for $n$ even \\
$\left(\mathfrak{s} o^{*}(2 n), \mathfrak{s} o(n, \mathbb{C}), \mathfrak{s} u(n, n)\right)$ & 2 for $n$ odd \\
$(\mathfrak{s} p(2 n, \mathbb{R}), \mathfrak{s} p(n, \mathbb{C}), \mathfrak{s} u(2 n, 2 n))$ & 1 \\
$(\mathfrak{s o}(2, q) \oplus \mathfrak{s} o(p+1), \mathfrak{s} o(1, q) \oplus \mathfrak{o}(p)$, & 1 for $n \equiv 3(\bmod 4)$ \\
$\mathfrak{s} o(2, n+1))$ & 2 for $n \equiv 1(\bmod 4)$ \\
$(\mathfrak{g}, \mathfrak{h}, \mathfrak{g} \times \mathfrak{g})$, for $(\mathfrak{g}, \mathfrak{h})$ of Cayley type & 4 for $n$ even \\
& 2 for $\mathfrak{g} \neq \mathfrak{s} o(2,2 k+1), \mathfrak{s} p(2 n, \mathbb{R})$ \\
$(\mathfrak{s} o(2, n), \mathfrak{s} o(1, n), \mathfrak{s} o(2, n+1))$ & 1 for $n$ odd \\
& 2 for $n$ even \\
\hline
\end{tabular}

For $G_{1}$ we define $P_{1}^{ \pm}, K_{1 \mathbb{C}}$, and the functions $p_{1}^{ \pm}$and $k_{1 \mathbb{C}}$ in the same manner as for $G$. Since the map $P_{1}^{+} \times K_{1 \mathbb{C}} \times P_{1}^{-} \rightarrow G_{1 \mathbb{C}},\left(p^{+}, k, p^{-}\right) \mapsto$ $p^{+} k p^{-}$, is a diffeomorphism onto a dense subset we can define

$$
\zeta_{1}: P_{1}^{+} K_{1 \mathbb{C}} P_{1}^{-} \rightarrow \mathfrak{p}_{1}^{+} \quad g \mapsto \exp ^{-1} p_{1}^{+}(g) .
$$

We will use the Harish-Chandra realization $D_{1}:=\zeta_{1}\left(G K_{1 \mathbb{C}} P_{1}^{-}\right)$of $G_{1} / K_{1}$ in $\mathfrak{p}_{1}^{+}$and $\zeta_{1}$ to state our results in a particularly nice form and note therefore:

Proposition 4.2. In the Harish-Chandra realization $\check{S}_{1}=\zeta_{1}\left(G_{1} c\right)$ and the image $\Phi(G / H)$ of the causal compactification is given by $\zeta_{1}(G c)$.

Proof. The first assertion is [KW65, Theorem 3.6], the second then follows from Theorem 2.3.

To avoid a clumsy notation we will assume from now on, using the Proposition, that $\Phi(G / H) \subset \mathfrak{p}_{1}^{+}$. Define the canonical cocycle

$$
J_{1}(g, z)=J_{1}^{1}(g, z):=k_{1 \mathbb{C}}(g \exp z)^{2 \rho_{1, n}},
$$


where $(\cdot)^{2 \rho_{1, n}}$ is the central character of $K_{1 \mathbb{C}}$ defined by $2 \rho_{1, n}$. More general, assume $m \geq 1$ such that $\left(\pi_{m}, V_{m}\right)$ exists then

$$
J_{1}^{m / 2}(g, z):=k_{1 \mathbb{C}}(g \exp z)^{m \rho_{1, n}}
$$

is defined. If $\left(\pi_{m}, V_{m}\right)$ is $G$-spherical define

$$
\psi_{m}: \mathfrak{p}_{1}^{+} \rightarrow \mathbb{C} \quad z \mapsto\left(\pi_{m}\left(\exp (z) c^{-1}\right) v_{m} \mid \xi_{m}\right) .
$$

Theorem 4.3. Assume $m \geq 1$ such that $\left(\pi_{m}, V_{m}\right)$ exists and is $G$-spherical. Then the function $\psi_{m}(z)$ is holomorphic on $\mathfrak{p}_{1}^{+}$and has the following properties:

(1) $\Phi(G / H)=\left\{z \in \check{S}_{1} \mid \psi_{m}(z) \neq 0\right\}$.

(2) For $g \in G_{\mathbb{C}}, z \in \mathfrak{p}_{1}^{+}$, such that $g \cdot z$ is defined, we have

$$
\psi_{m}(g \cdot z)=J_{1}^{m / 2}(g, z) \psi_{m}(z) .
$$

Proof. (1) For $z=\zeta_{1}(g c) \in \check{S}_{1}$, with $g \in G_{1}$, we have

$$
\begin{aligned}
\psi_{m}(z) & =\left(\pi_{m}\left(p_{1}^{+}(g c) c^{-1}\right) v_{m} \mid \xi_{m}\right) \\
& =\left(\pi_{m}\left(g c p_{1}^{-}(g c)^{-1} k_{1 \mathbb{C}}(g c)^{-1} c^{-1}\right) v_{m} \mid \xi_{m}\right) .
\end{aligned}
$$

Since $\operatorname{Ad}(c) i Z^{0}=\frac{1}{2} X^{0}$, the Cayley transformed group $\operatorname{Ad}(c)\left(K_{1 \mathbb{C}} P_{1}^{-}\right)$is $P_{\mathbb{C}}^{\prime}$, where we denote by $\operatorname{Ad}(c)$ the inner automorphism $x \mapsto c x c^{-1}$ of $G_{1 \mathbb{C}}$. In [B97] it is shown that $G P^{\prime}$ is the only open $\left(G, P^{\prime}\right)$-double coset in $G_{1}$. Then by [BD92, Lemme 4] we have $\left(\pi_{m}(g p) v_{m} \mid \xi_{m}\right) \neq 0$, for $p \in P_{\mathbb{C}}^{\prime}$, if and only if $g \in G$. (As $M^{\prime} / M_{0}^{\prime}$ is finite, and using that every component contains an element of the form $\exp i \lambda X^{0}$, the cited result is still valid with obvious modifications in its proof, even if $v_{m}$ is not $M_{\mathbb{C}}^{\prime}$-fixed. Note also, that we use a lowest weight vector $v_{m}$, since the nilradical $\mathfrak{q}_{1 \eta}^{-}$of our parabolic algebra $\mathfrak{p}^{\prime}$ is the sum of root spaces for negative roots.)

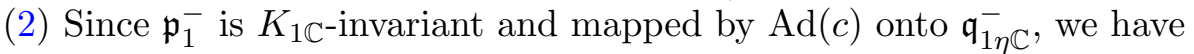

$$
\begin{aligned}
& \psi_{m}(g \cdot z) \\
& =\left(\pi_{m}\left(g \exp z p_{1}^{-}(g \exp z)^{-1} k_{1 \mathbb{C}}(g \exp z)^{-1} c^{-1}\right) v_{m} \mid \xi_{m}\right) \\
& =\left(\pi_{m}\left(\exp z p_{1}^{-}(g \exp z)^{-1} k_{1 \mathbb{C}}(g \exp z)^{-1} c^{-1}\right) v_{m} \mid \pi_{m}\left(\theta(g)^{-1}\right) \xi_{m}\right) \\
& =\left(\pi_{m}\left(\exp z k_{1 \mathbb{C}}(g \exp z)^{-1} c^{-1} \operatorname{Ad}\left(c k_{1 \mathbb{C}}(g \exp z)\right)\left(p_{1}^{-}(g \exp z)^{-1}\right)\right) v_{m} \mid \xi_{m}\right) \\
& =\left(\pi_{m}\left(\exp z c^{-1} \operatorname{Ad}(c)\left(k_{1 \mathbb{C}}(g \exp z)^{-1}\right)\right) v_{m} \mid \xi_{m}\right) \\
& =J_{1}^{m / 2}(g, z)\left(\pi_{m}\left(\exp z c^{-1}\right) v_{m} \mid \xi_{m}\right) .
\end{aligned}
$$

Example. We want to describe the causal compactification for $G / H$ of Cayley type. Let $G_{1}=\widetilde{G} \times \widetilde{G}$, with $\widetilde{G} \subset \widetilde{G}_{\mathbb{C}}$ simply connected and $(\widetilde{\mathfrak{g}}, \widetilde{\mathfrak{k}}, \widetilde{\theta})$ Hermitian symmetric of tube type. With $\sigma(g, h):=(h, g)$ the fixpoint group $G$ of this involution is isomorphic to $\widetilde{G}$. For $\widetilde{\mathfrak{a}}_{p} \subset \widetilde{\mathfrak{p}}$ maximal abelian and 
$\theta=\widetilde{\theta} \times \widetilde{\theta}$, we have $\mathfrak{a}_{p}=\widetilde{\mathfrak{a}}_{p} \times \widetilde{\mathfrak{a}}_{p}$ and $\mathfrak{b}=\left\{(X,-X) \mid X \in \widetilde{\mathfrak{a}}_{p}\right\}$. With $\widetilde{\Delta}:=$ $\Delta\left(\widetilde{\mathfrak{g}}, \widetilde{\mathfrak{a}}_{p}\right)$ we can define $\widetilde{\rho}_{+}$and get then $\rho_{+}=\left(\widetilde{\rho}_{+},-\widetilde{\rho}_{+}\right)$. Given $\left(\widetilde{\pi}_{m}, \widetilde{V}_{m}\right)$, a representation of $\widetilde{G}_{\mathbb{C}}$ with lowest weight $-m \widetilde{\rho}_{+}$, the representation $\pi_{m}:=$ $\tilde{\pi}_{m} \otimes \tilde{\pi}_{m}^{*}$, where $\tilde{\pi}_{m}^{*}$ is the contragredient representation to $\widetilde{\pi}_{m}$, has lowest weight $-m \rho_{+}$. Moreover every such representation has the $G$-fixed vector $\sum w_{i} \otimes w_{i}^{*}$, where $\left\{w_{i} \mid i=1, \ldots, \operatorname{dim} \widetilde{V}_{m}\right\}$ is a base of $\widetilde{V}_{m}$ and $\left\{w_{i}^{*}\right\}$ its dual. We have now $c=\left(\widetilde{c}, \widetilde{c}^{-1}\right)$ and for $(z, w) \in \mathfrak{p}_{1}^{+}$

$$
\begin{aligned}
\psi_{m}((z, w)) & =\left(\widetilde{\pi}_{m}\left(\exp z \widetilde{c}^{-1}\right) \otimes \widetilde{\pi}_{m}^{*}(\exp w \widetilde{c}) \cdot \widetilde{v}_{m} \otimes \widetilde{v}_{m}^{*} \mid \sum w_{i} \otimes w_{i}^{*}\right) \\
& =\sum\left(\widetilde{\pi}_{m}\left((\exp w \widetilde{c})^{-1} \exp z \widetilde{c}^{-1}\right) \otimes 1 \cdot \widetilde{v}_{m} \otimes \widetilde{v}_{m}^{*} \mid w_{i} \otimes w_{i}^{*}\right) \\
& =\left(\pi_{m}\left(\widetilde{c}^{-1} \exp (z-w) \widetilde{c}^{-1}\right) v_{m} \mid v_{m}\right) \\
& =\left(\pi_{m}\left(\widetilde{c}^{-2} \exp (z-w)\right) \widetilde{\pi}_{m}\left(\widetilde{c}^{-1}\right) v_{m} \mid \widetilde{\pi}_{m}\left(\widetilde{c}^{-1}\right) v_{m}\right) .
\end{aligned}
$$

Here we used $\widetilde{\mathfrak{t}}^{+}+\widetilde{\mathfrak{a}}_{p}$ as a Cartan algebra contrary to $\widetilde{\mathfrak{t}}^{+}+\widetilde{\mathfrak{t}}^{-}$used in [ÓØ99]. Therefore the lowest weight vectors are related by the Cayley transform and our $\psi_{m}$ is the same function as those constructed in [ÓØ99].

\section{Some $L^{2}$-isometries.}

We know that $\Phi(G / H) \subset \check{S}_{1}$ is open and dense. In this section we relate the $L^{2}$-spaces with respect to invariant measures on these manifolds. The following results can be found in [ÓØ99]:

For $\check{S}_{1}$ in the Harish-Chandra realization the quasi-invariant measure is given by

$$
\int_{\check{S}_{1}} f(z) d \mu(z)=\int_{K_{1} / K_{1} \cap Z_{G_{1}}\left(X^{0}\right)} f\left(k \cdot \zeta_{1}(c)\right) d k .
$$

With $P^{\prime}=Z_{G_{1}}\left(X^{0}\right) Q_{1 \eta}^{-}$we can decompose every $g \in G_{1}$ in the form

$$
g=k_{1}(g) h(g) q^{-}(g),
$$

where $k_{1}(g) \in K_{1}, h(g) \in Z_{G_{1}}\left(X^{0}\right)$, and $q^{-}(g) \in Q_{1 \eta}^{-}$. To $z \in \check{S}_{1}$ choose $k \in K_{1}$ such that $z=k \cdot \zeta_{1}(c)$ and define

$$
J_{R}(g, z):=h(g k)^{2 \rho_{+}}=\operatorname{det}\left(\left.\operatorname{Ad}(h(g k))\right|_{q_{1 \eta}^{-}}\right)^{-1},
$$

then

$$
\int_{\check{S}_{1}} f(g \cdot z) J_{R}(g, z) d \mu(z)=\int_{\check{S}_{1}} f(z) d \mu(z)
$$

and the relation

$$
k_{1 \mathbb{C}}(g \exp z)^{2 \rho_{1, n}}=k_{1}(g k)^{2 \rho_{1, n}} k^{-2 \rho_{1, n}} h(g k)^{2 \rho_{+}}
$$

holds. Note that the two first factors on the right hand side have modulus one by the compactness of $K_{1}$. 
For $k_{1 \mathbb{C}}$ we also have the cocycle property

$$
k_{1 \mathbb{C}}(g h \exp z)=k_{1 \mathbb{C}}(g \exp (h \cdot z)) k_{1 \mathbb{C}}(h \exp z),
$$

which gives

$$
J_{1}^{m / 2}(g h, z)=J_{1}^{m / 2}(g, h \cdot z) J_{1}^{m / 2}(h, z)
$$

when $J_{1}^{m / 2}$ is defined. The following theorem now follows from the cocycle property for $J_{1}(\cdot, \cdot)$.

Theorem 5.1. (1) The G-invariant measure on $\Phi(G / H) \subset \check{S}_{1}$ is up to normalization given by

$$
\int_{\check{S}_{1}} f(z)\left|\psi_{m}(z)\right|^{-2 / m} d \mu(z)
$$

(2) A unitary representation of $G_{1}$ in $L^{2}\left(\check{S}_{1}\right)$ is given by

$$
\left(\lambda_{0}(g) f\right)(z):=\sqrt{\left|J_{1}\left(g^{-1}, z\right)\right|} f\left(g^{-1} \cdot z\right) .
$$

(3) Identify $G / H$ with $\zeta_{1}(\Phi(G / H))$ and let $\lambda$ denote the left regular representation on $G / H$, then $f \mapsto f\left|\psi_{m}\right|^{1 / m}$ is a $G$-equivariant isometry of $\left(L^{2}\left(\check{S}_{1}\right), \lambda_{0} \mid G\right)$ onto $\left(L^{2}(G / H), \lambda\right)$.

If $\mathfrak{g}_{1} \neq \mathfrak{s} p(2 n, \mathbb{R}), \mathfrak{s} o(2,2 k+1)$, the holomorphic square root $\sqrt{J_{1}}=J_{1}^{1 / 2}$ is well-defined. We get then in the same way as the preceding theorem.

Theorem 5.2. (1) If $\mathfrak{g}_{1} \neq \mathfrak{s} p(2 n, \mathbb{R}), \mathfrak{s} o(2,2 k+1)$, then

$$
\left(\lambda_{1}(g) f\right)(z):=\sqrt{J_{1}\left(g^{-1}, z\right)} f\left(g^{-1} \cdot z\right)
$$

is a unitary representation of $G_{1}$ in $L^{2}\left(\check{S}_{1}\right)$.

(2) If further $\pi_{1}$ is $G$-spherical then $f \mapsto f \psi_{1}$ is a $G$-equivariant isometry of $\left(L^{2}\left(\check{S}_{1}\right), \lambda_{1} \mid G\right)$ onto $\left(L^{2}(G / H), \lambda\right)$.

In the general case, to define the roots $\sqrt{J_{1}(\cdot, \cdot)}$ and $\sqrt[m]{\psi_{m}}$, we have to replace $G_{1}$ and $\Phi(G / H)$ by appropriate coverings. For

$$
\mathcal{D}:=\left\{z \in \bar{D}_{1} \mid \psi_{m}(z) \neq 0\right\},
$$

which is independent of $m$ by the last section, define a $m$-fold covering by

$$
\widetilde{\mathcal{D}}:=\left\{(z, x) \in \mathcal{D} \times \mathbb{C} \mid \psi_{m}(z)=x^{m}\right\} .
$$

We define then a holomorphic $m$-th root $\sqrt[m]{\psi_{m}}$ on $\widetilde{\mathcal{D}}$ by

$$
\sqrt[m]{\psi_{m}}: \widetilde{\mathcal{D}} \rightarrow \mathbb{C} \quad(z, x) \mapsto x .
$$

In the same manner we define a double covering $\widetilde{G}_{1}$ of $G_{1}$ such that $\sqrt{J_{1}(\cdot, \cdot)}$ is defined on $\widetilde{G}_{1} \times \bar{D}_{1}$ and equal to 1 on $\left[\widetilde{K}_{1}, \widetilde{K}_{1}\right] \times\{0\}$. More general, as the Ol'shanskil semigroup $\Gamma_{1}\left(W_{k}\right)$ is homeomorphic to $G_{1} \times i W_{k}$ and $\Gamma_{1}\left(W_{k}\right) \subset\left\{\gamma \in G_{1 \mathbb{C}} \mid \gamma^{-1} \bar{D}_{1} \subset \bar{D}_{1}\right\}$ ([HÓØ91]), there is a double 
covering $\widetilde{\Gamma}_{1}\left(W_{k}\right)$ with $\sqrt{J_{1}(\cdot, \cdot)}$ defined on $\widetilde{\Gamma}_{1}\left(-W_{k}\right) \times \bar{D}_{1}$ and holomorphic on $\widetilde{\Gamma}_{1}\left(-W_{k}\right)^{o} \times D_{1}$.

Let $\kappa: \widetilde{\Gamma}_{1}\left(-W_{k}\right) \rightarrow \Gamma_{1}\left(-W_{k}\right)$ be the canonical projection, then the covering semigroup operates on $\bar{D}_{1}$ by projecting first with $\kappa$. Moreover the cocycle relation

$$
\sqrt{J_{1}(\widetilde{\gamma} \widetilde{\delta}, z)}=\sqrt{J_{1}(\widetilde{\gamma}, \widetilde{\delta} \cdot z)} \sqrt{J_{1}(\widetilde{\delta}, z)}
$$

with $\widetilde{\gamma}, \widetilde{\delta} \in \widetilde{\Gamma}_{1}\left(-W_{k}\right)$ and $z \in \bar{D}_{1}$ is still valid. Let $\widetilde{\Gamma}( \pm W):=\kappa^{-1}(\Gamma( \pm W)) \subset$ $\widetilde{\Gamma}_{1}\left( \pm W_{k}\right)$ be the set lying above $\Gamma( \pm W)$ then by Theorem 4.3 we get the $\Gamma(-W)$ - respectively $\widetilde{\Gamma}(-W)$-invariance of $\mathcal{D} \subset \bar{D}_{1}$. We have an operation of $\widetilde{\Gamma}(-W)$ respectively $\widetilde{G}$ on $\widetilde{\mathcal{D}}$ by setting

$$
\widetilde{\gamma} \cdot(z, x):=\left(\widetilde{\gamma} \cdot z, \sqrt{J_{1}(\widetilde{\gamma}, z)} x\right) .
$$

Therefore we can define as usual the left action $\tilde{\lambda}$ by

$$
\widetilde{\lambda}(\widetilde{\gamma}) f(z, x):=f\left(\widetilde{\gamma}^{-1} \cdot(z, x)\right)
$$

for functions defined on $\widetilde{\mathcal{D}}$.

For $X \subset \mathcal{D}$ let $\widetilde{X} \subset \widetilde{\mathcal{D}}$ be the subset lying above $X$.

Definition. Let $X \subset \mathcal{D}$ and $f: \widetilde{X} \rightarrow \mathbb{C}$ be a function.

(1) The function $f$ is odd if $f(z, \xi x)=\xi f(z, x)$ for all $m$-th roots of unity $\xi$ and $(z, x) \in \widetilde{X}$.

(2) The function $f$ is even if $f(z, \xi x)=f(z, x)$ for all $m$-th roots of unity $\xi$ and $(z, x) \in \widetilde{X}$.

Every even function corresponds to a unique function on $X$ and, by abuse of notation, we will denote both by the the same letter. We note also that we have a $\widetilde{G}$-invariant measure on $\widetilde{G / H}$, defined as the pullback of the $G$-invariant measure on $G / H$ (and normalized by $m^{-1}$ ). Therefore the $L^{2}$ space of odd functions $L_{\text {odd }}^{2}(\widehat{G / H})$ is a well-defined object.

With these definitions the proof of the last theorem can be generalized now and we get:

Theorem 5.3. (1) We have a unitary representation of $\widetilde{G}_{1}$ in $L^{2}\left(\check{S}_{1}\right)$ given by

$$
\left(\widetilde{\lambda}_{1}(\widetilde{g}) f\right)(z):=\sqrt{J_{1}\left(\widetilde{g}^{-1}, z\right)} f\left(\widetilde{g}^{-1} \cdot z\right) .
$$

(2) If $\pi_{m}$ is G-spherical then $L^{2}\left(\check{S}_{1},\left.\widetilde{\lambda}_{1}\right|_{\widetilde{G}}\right) \rightarrow L_{\text {odd }}^{2}(\widetilde{G / H}, \widetilde{\lambda}), f \mapsto f \sqrt[m]{\psi_{m}}$, is a surjective $\widetilde{G}$-equivariant isometry. 


\section{Characterization of $\Phi\left(\Xi\left(C^{0}\right)\right)$.}

Remember that $D_{1}=\zeta_{1}\left(G_{1} K_{1 \mathbb{C}} P_{1}^{-}\right)$is the Hermitian symmetric space in the Harish-Chandra realization. Since $\mathfrak{b} \subset \mathfrak{q}_{1 \sigma} \cap \mathfrak{p}_{1}$ is maximal abelian, we have $G_{1}=G \exp (\mathfrak{b}) K_{1}$. This, together with Proposition 2.4(1), gives now

$$
D_{1}=G \cdot\left\{\sum x_{j^{\prime}} E_{j^{\prime}}^{\prime}|| x_{j^{\prime}} \mid<1\right\}
$$

by a $\mathfrak{s l}(2)$-calculation, cf. [H78, p. 387].

Proposition 6.1. (1) $\Phi\left(\Xi\left(C^{o}\right)\right)=\left\{z \in D_{1} \mid \psi_{m}(z) \neq 0\right\}$.

(2) There is a constant $c \neq 0$ such that

$$
\psi_{m}\left(\sum x_{j^{\prime}} E_{j^{\prime}}^{\prime}\right)=c \prod_{j^{\prime}} x_{j^{\prime}}^{m \rho_{1, n}\left(H_{j^{\prime}}^{\prime} / 2\right)}=c \prod_{j^{\prime}} x_{j^{\prime}}^{\frac{m}{2}\left(1+\frac{d(r-1)}{2}\right) \sum_{j} \lambda_{j^{\prime}, j}} .
$$

Proof. First we note that $\Gamma\left(-W^{o}\right) Z_{G_{1 \mathbb{C}}}\left(X^{0}\right)=G \exp \left(-i C^{o}\right) Z_{G_{1 \mathbb{C}}}\left(X^{0}\right)$, which can be proven as in [HÓØ91, Prop. 1.4]. Using this, we get

$$
\Phi\left(\Xi\left(C^{o}\right)\right)=\zeta_{1}\left(\Gamma(-W)^{o} \cdot c\right)=\zeta_{1}\left(G \exp \left(-i\left(\mathfrak{a} \cap C^{o}\right)\right) \cdot c\right),
$$

since $H \subset Z_{G_{1 \mathbb{C}}}\left(X^{0}\right) \subset \operatorname{Stab}_{G_{1 \mathbb{C}}}(c)=P_{\mathbb{C}}^{\prime}$ and using Proposition 2.4(5). By a $\mathfrak{s l}(2)$-calculation

$$
(* *) \quad \exp \left(\lambda H_{j}\right) \cdot E_{k}=\left\{\begin{array}{ll}
E_{k}, & \text { if } j \neq k \\
e^{2 \lambda} E_{k} & \text { if } j=k
\end{array},\right.
$$

and, as $\zeta_{1}(c)=-\sum E_{j}=-\sum E_{j^{\prime}}^{\prime}$, we finally get from Proposition 2.4(1) and (6) that

$$
\Phi\left(\Xi\left(C^{o}\right)\right)=G \cdot\left\{\sum x_{j^{\prime}} E_{j^{\prime}}^{\prime}|0<| x_{j^{\prime}} \mid<1\right\} \subset D_{1} .
$$

Conversely, to determine the set $\left\{z \in D_{1} \mid \psi_{m}(z) \neq 0\right\}$ by Theorem 4.3 it is enough to consider $\left\{\psi_{m}\left(\sum x_{j^{\prime}} E_{j^{\prime}}^{\prime}\right)|| x_{j^{\prime}} \mid<1\right\}$. Using Proposition 2.4(1) we can calculate $\psi_{m}\left(\sum x_{j^{\prime}} E_{j^{\prime}}^{\prime}\right)$ via $\mathfrak{s l}(2)$-reductions. Thus we assume for the moment $G_{1 \mathbb{C}}=S L(2, \mathbb{C})$,

$$
E_{j^{\prime}}^{\prime}=\left(\begin{array}{cc}
0 & 1 \\
0 & 0
\end{array}\right), E_{-j^{\prime}}^{\prime}=\left(\begin{array}{cc}
0 & 0 \\
1 & 0
\end{array}\right), \text { and } H_{j^{\prime}}^{\prime}=\left(\begin{array}{cc}
1 & 0 \\
0 & -1
\end{array}\right)
$$

for $j^{\prime}$ fixed. Then $G_{1 \mathbb{C}}^{\sigma} \exp \left(\mathbb{C} X_{j^{\prime}}^{\prime}\right) Q_{1 \eta \mathbb{C}}^{-} \subset G_{1 \mathbb{C}}$ is open and dense, defining a $r_{j^{\prime}} \in \mathbb{C}$ by $\exp \left(x_{j^{\prime}} E_{j^{\prime}}^{\prime}\right) c^{-1} \in G_{1 \mathbb{C}}^{\sigma} \exp \left(r_{j^{\prime}} X_{j^{\prime}}^{\prime}\right) Q_{1 \eta \mathbb{C}}^{-}$for almost all $x_{j^{\prime}}$. To determine $r_{j^{\prime}}$, choose $(X \mid Y):=\operatorname{tr}\left(X Y^{*}\right)$ as an invariant scalar product on $\mathfrak{g}_{1 \mathbb{C}}$ and $v \in \mathfrak{q}_{1 \eta \mathbb{C}}^{+}$nonzero. Let $g \in G_{1 \mathbb{C}}^{\sigma}$ and $q \in Q_{1 \eta \mathbb{C}}^{-}$be chosen such that $\exp \left(x_{j^{\prime}} E_{j^{\prime}}^{\prime}\right) c^{-1}=g \exp \left(r_{j^{\prime}} X_{j^{\prime}}^{\prime}\right) q$. Then we have

$$
\left(\exp \left(x_{j^{\prime}} E_{j^{\prime}}^{\prime}\right) c^{-1}\right)^{-1} \sigma\left(\exp \left(x_{j^{\prime}} E_{j^{\prime}}^{\prime}\right) c^{-1}\right)=q^{-1} \exp \left(-2 x_{j^{\prime}} X_{j^{\prime}}^{\prime}\right) \sigma(q)
$$


and consequently, using $\sigma\left(Q_{1 \eta \mathbb{C}}^{-}\right)=\theta\left(Q_{1 \eta \mathbb{C}}^{-}\right)=Q_{1 \eta \mathbb{C}}^{+}$, we get

$$
\left(\operatorname{Ad}\left(\left(\exp \left(x_{j^{\prime}} E_{j^{\prime}}^{\prime}\right) c^{-1}\right)^{-1} \sigma\left(\exp \left(x_{j^{\prime}} E_{j^{\prime}}^{\prime}\right) c^{-1}\right)\right) v \mid v\right)=e^{-4 r_{j^{\prime}}}(v \mid v) .
$$

Inserting the appropriate matrices it follows easily $r_{j^{\prime}}=-\frac{1}{2} \log 2 x_{j^{\prime}}$ and further

$$
\psi_{m}\left(\sum x_{j^{\prime}} E_{j^{\prime}}^{\prime}\right)=\left(\prod_{j^{\prime}} e^{-m \rho_{+}\left(r_{j^{\prime}} X_{j^{\prime}}^{\prime}\right)}\right)\left(v_{m} \mid \xi_{m}\right)=c \prod_{j^{\prime}} x_{j^{\prime}}^{\frac{m}{2}\left(1+\frac{d(r-1)}{2}\right) \sum_{j} \lambda_{j^{\prime}, j}}
$$

Remark. Using $C=\operatorname{Ad}(H)(\mathfrak{a} \cap C)$ together with $\mathfrak{a} \cap C=-\sum \mathbb{R}_{0}^{+} i H_{j^{\prime}}^{\prime}$ the arguments of the proof show that $\Xi(C) \subset \mathcal{D}$.

\section{Some Hardy space isometries.}

The classical Hardy space for $G_{1}$ respectively the covering group $\widetilde{G}_{1}$ in case $\mathfrak{g}_{1}=\mathfrak{s} p(2 n, \mathbb{R}), \mathfrak{s} o(2,2 k+1)$, is defined as the Hilbert space

$$
\begin{aligned}
H_{c l}:=\left\{f: D_{1} \rightarrow \mathbb{C} \mid f\right. \text { is holomorphic and } \\
\left.\qquad \sup _{\{0<r<1\}} \int_{K_{1}}|f(r k \cdot c)|^{2} d k<\infty\right\}
\end{aligned}
$$

with $G_{1}$-action

$$
(\lambda(g) f)(z):=\sqrt{J_{1}\left(g^{-1}, z\right)} f\left(g^{-1} \cdot z\right)
$$

respectively $\widetilde{G}_{1}$-action

$$
(\widetilde{\lambda}(\widetilde{g}) f)(z):=\sqrt{J_{1}\left(\widetilde{g}^{-1}, z\right)} f\left(\widetilde{g}^{-1} \cdot z\right)
$$

([FK94, XIII,3]). By Theorem 5.2(1) respectively Theorem 5.3(1), the operation of taking boundary values is a $G_{1}$ - respectively $\widetilde{G}_{1}$-equivariant isometry into $L^{2}\left(\breve{S}_{1}\right)$.

Theorem 7.1. We identify $\Xi\left(C^{o}\right)$ with $\Phi\left(\Xi\left(C^{o}\right)\right)$. If $\mathfrak{g}_{1} \neq \mathfrak{s} p(2 n, \mathbb{R})$, $\mathfrak{s o}(2,2 k+1)$ and $\pi_{1}$ is $G$-spherical then $I:\left.H_{c l} \rightarrow H_{2}(C) f \mapsto f \psi_{1}\right|_{\Xi\left(C^{o}\right)}$ is a $G$-equivariant isometry.

Proof. In $H_{2}(C)$ the action is given by left translation, i.e., for $f \in H_{c l}$ we get

$$
\begin{aligned}
(\lambda(g) I(f))(z) & =f\left(g^{-1} \cdot z\right) \psi_{1}\left(g^{-1} \cdot z\right) \\
& =\sqrt{J_{1}\left(g^{-1}, z\right)} f\left(g^{-1} \cdot z\right) \psi_{1}(z)=I(\lambda(g) f)(z),
\end{aligned}
$$

the second equality by Theorem 4.3. The map is also an isometry by Theorem 5.2(2), as taking boundary values is an isometry for the Hardy spaces. 
For the general case we define first $\widetilde{H}_{2}(C)$ as covering space of $H_{2}(C)$ with a $\widetilde{\Gamma}(W)$-action by

$$
\widetilde{H}_{2}(C):=\left\{f: \widetilde{\Xi}\left(C^{o}\right) \rightarrow \mathbb{C} \mid f \text { is holomorphic and }\|f\|_{\widetilde{H}}<\infty\right\},
$$

where $\|f\|_{\widetilde{H}}:=\sup _{\widetilde{\gamma} \in \widetilde{\Gamma}^{0}}\|\widetilde{\gamma} \cdot f\|_{L^{2}(\widetilde{G / H})}$. For this space the analogous results as stated in Section 3 hold. One proves now in the same manner as the theorem above:

Theorem 7.2. If $\pi_{m}$ is $G$-spherical then

$$
\widetilde{I}:\left.H_{c l} \rightarrow \widetilde{H}_{2, \text { odd }}(C) f \mapsto f \sqrt[m]{\psi_{m}}\right|_{\widetilde{\Xi}\left(C^{o}\right)}
$$

is a $\widetilde{G}$-equivariant isometry.

Let us look closer at the different cases $m=1,2,4$.

$\mathbf{m}=1$ : In this case both $\psi_{1}$ and $\sqrt{J_{1}}$ exists, so all coverings split in a trivial way. In particular $L_{\text {odd }}^{2}(\widetilde{G / H}) \simeq L_{\text {even }}^{2}(\widetilde{G / H}) \simeq L^{2}(G / H)$ and similarly also $\widetilde{H}_{2, \text { odd }}(C) \simeq \widetilde{H}_{2 \text {,even }}(C) \simeq H_{2}(C)$. By the table before Proposition 4.2 the following $\left(\mathfrak{g}, \mathfrak{h}, \mathfrak{g}_{1}\right)$-triples correspond to this case:

\begin{tabular}{|l|l|}
\hline$(\mathfrak{s}(\mathfrak{u}(p, q) \oplus \mathfrak{u}(p, q)), \mathfrak{s} u(p, q), \mathfrak{s} u(n, n))$ & $n$ even \\
$(\mathfrak{u}(p, q), \mathfrak{s} o(p, q), \mathfrak{s} p(n, \mathbb{R}))$ & $n=3(\bmod 4)$ \\
$(\mathfrak{s} o(2, n-1) \oplus \mathfrak{s} o(2), \mathfrak{s} o(1, n-1), \mathfrak{s} o(2, n+1))$ & $n=3(\bmod 4)$ \\
$\left(\mathfrak{s} o^{*}(2 n), \mathfrak{s} o(n, \mathbb{C}), \mathfrak{s} u(n, n)\right)$ & $n$ even \\
$(\mathfrak{s} p(2 n, \mathbb{R}), \mathfrak{s} p(n, \mathbb{C}), \mathfrak{s} u(2 n, 2 n))$ & \\
$(\mathfrak{s} o(2, q) \oplus \mathfrak{s} o(p+1), \mathfrak{s} o(1, q) \oplus \mathfrak{o}(p), \mathfrak{s} o(2, n+1))$ & $n \equiv 3(\bmod 4)$ \\
$(\mathfrak{g}, \mathfrak{h}, \mathfrak{g} \times \mathfrak{g})$ for $(\mathfrak{g}, \mathfrak{h})$ of Cayley type & $\mathfrak{g} \neq \mathfrak{s} o(2,2 k+1)$, \\
& $\mathfrak{s} p(2 n, \mathbb{R})$ \\
$(\mathfrak{s} o(2, n), \mathfrak{s} o(1, n), \mathfrak{s} o(2, n+1))$ & $n$ odd \\
\hline
\end{tabular}

$\mathbf{m}=\mathbf{2}$ : Let us first assume $\mathfrak{g}_{1} \neq \mathfrak{s} p(2 n, \mathbb{R}), \mathfrak{s o}(2,2 k+1)$ such that $\sqrt{J_{1}}$ exists as a holomorphic function on $\Gamma_{1}\left(-W_{k}^{o}\right) \times D_{1}$. Those are the $\left(\mathfrak{g}, \mathfrak{h}, \mathfrak{g}_{1}\right)$-triples:

\begin{tabular}{|l|l|}
\hline$(\mathfrak{s}(\mathfrak{u}(p, q) \oplus \mathfrak{u}(p, q)), \mathfrak{s} u(p, q), \mathfrak{s} u(n, n))$ & $n$ odd \\
$\left(\mathfrak{u}(2 p, 2 q), \mathfrak{s} p(p, q), \mathfrak{s} o^{*}(4 n)\right)$ & \\
$(\mathfrak{u}(p, q), \mathfrak{s} o(p, q), \mathfrak{s} p(n, \mathbb{R}))$ & $n=1(\bmod 4)$ \\
$(\mathfrak{s} o(2, n-1) \oplus \mathfrak{s} o(2), \mathfrak{s} o(1, n-1), \mathfrak{s} o(2, n+1))$ & $n=1(\bmod 4)$ \\
$\left(\mathfrak{e}_{6}(-14) \oplus \mathfrak{s} o(2), \mathfrak{f}_{\left.4(-20), \mathfrak{e}_{7}(-25)\right)}\right.$ & \\
$\left(\mathfrak{s} o^{*}(2 n) \oplus \mathfrak{s} o^{*}(2 n), \mathfrak{s} o^{*}(2 n), \mathfrak{s} o^{*}(4 n)\right)$ & \\
$\left(\mathfrak{s} o^{*}(2 n), \mathfrak{s} o(n, \mathbb{C}), \mathfrak{s} u(n, n)\right)$ & $n$ odd \\
$(\mathfrak{s} o(2, q) \oplus \mathfrak{s} o(p+1), \mathfrak{s} o(1, q) \oplus \mathfrak{o}(p), \mathfrak{s} o(2, n+1))$ & $n \equiv 1(\bmod 4)$ \\
\hline
\end{tabular}

Let $E:=\sum E_{j}=-\zeta_{1}(c)$. By the equation

$$
\psi_{2}(g \cdot z)=J_{1}(g, z) \psi_{2}(z)
$$


and the fact that $\Gamma(-W)$ acts transitively on $\Xi(C)$ we can define a global square root of $\psi_{2}$ on $\Xi(C)$ by

$$
\sqrt{\psi_{2}}(z):=\sqrt{J_{1}\left(\gamma^{-1},-E\right)}
$$

with $\gamma$ chosen such that $z=\gamma^{-1} \cdot(-E)$. Clearly this square root is holomorphic on $\Xi\left(C^{o}\right)$.

Lemma 7.3. For

$$
\Xi(C)_{+}:=\left\{\left(\gamma \cdot(-E), \sqrt{J_{1}(\gamma,-E)}\right) \mid \gamma \in \Gamma(-W)\right\}
$$

and

we have:

$$
\Xi\left(C^{o}\right)_{-}:=\left\{\left(\gamma \cdot(-E),-\sqrt{J_{1}(\gamma,-E)}\right) \mid \gamma \in \Gamma(-W)\right\}
$$

(1) $\Xi(C)_{+}=\Gamma(-W) \cdot(-E, 1)$ and $\Xi(C)_{-}=\Gamma(-W) \cdot(-E,-1)$.

(2) $\widetilde{\Xi}(C)=\Xi(C)_{+} \dot{\cup} \Xi(C)_{-}$and the canonical projection $\kappa_{2}: \Xi(C)_{+} \rightarrow$ $\Xi(C)$ is a diffeomorphism, biholomorphic on $\Xi(C)_{+}^{o}$.

Proof. It is clear that $\Xi(C)_{+}, \Xi(C)_{-} \subset \widetilde{\Xi}(C)$ and that $\Xi(C)_{+} \cap \Xi(C)_{-}=\emptyset$. Let $\xi=(z, x) \in \widetilde{\Xi}(C)$ and $\gamma \in \Gamma(-W)$ such that $z=\gamma \cdot(-E)$ then

$$
x^{2}=\psi_{2}(z)=\psi_{2}(\gamma \cdot(-E))=J_{1}(\gamma,-E) \psi_{2}(-E)=J_{1}(\gamma,-E) .
$$

Thus $\xi=\left(z, \sqrt{J_{1}(\gamma,-E)}\right) \in \Xi(C)_{+}$or $\xi=\left(z,-\sqrt{J_{1}(\gamma,-E)}\right) \in \Xi(C)_{-}$and in particular $\Xi(C)=\Xi(C)_{+} \cup \Xi(C)_{-}$.

To define the inverse map to $\kappa_{2}$, choose for $z \in \Xi(C)$ a $\gamma \in \Gamma(-W)$ such that $z=\gamma \cdot(-E)$. Then $\kappa_{2}^{-1}$ is given by

$$
z \mapsto\left(z, \sqrt{J_{1}(\gamma,-E)}\right),
$$

which is a holomorphic map on $\Xi\left(C^{o}\right)=\Gamma\left(-W^{o}\right) \cdot(-E)$, since locally we may choose $\gamma$ using a holomorphic section.

The following is now clear:

Proposition 7.4. (1) The restriction from $\widetilde{\Xi}\left(C^{o}\right)$ to $\Xi(C)_{+}^{o}$ together with the canonical projection $\kappa_{2} \mid \Xi(C)_{+}^{o}: \Xi(C)_{+}^{o} \rightarrow \Xi\left(C^{o}\right)$ induces an isomorphism of $\Gamma(W)$-modules

$$
\widetilde{H}_{2, \text { odd }}(C) \simeq \widetilde{H}_{2, \text { even }}(C) \simeq H_{2}(C) .
$$

(2) The restriction from $\widetilde{G / H}$ to $G / H_{+}:=\widetilde{G / H} \cap \Xi(C)_{+}$together with the canonical projection $\kappa_{2} \mid G / H_{+}: G / H_{+} \rightarrow G / H$ induces an isomorphism of $G$-modules

$$
L_{\text {odd }}^{2}(\widetilde{G / H}) \simeq L_{\text {even }}^{2}(\widetilde{G / H}) \simeq L^{2}(G / H) .
$$

(3) Taking boundary values in the $L^{2}$-norm intertwines the G-actions on the respective modules. 
If $\left(\mathfrak{g}, \mathfrak{h}, \mathfrak{g}_{1}\right)=(\mathfrak{s o}(2,2 k), \mathfrak{s o}(1,2 k), \mathfrak{s o}(2,2 k+1))$ or $(\mathfrak{g}, \mathfrak{h}, \mathfrak{g} \times \mathfrak{g})$ is one of the remaining Cayley type cases the preceding construction does not work. However, using the fact that $\mathfrak{g}_{1}=\mathfrak{g} \times \mathfrak{g}$, for the Cayley type cases it is possible to define a global square root $\sqrt{J_{1}(\cdot, \cdot)}$ on $\Gamma(-W)$. Therefore analogous results to Lemma 7.3 and Proposition 7.4 hold, cf. [ÓØ99, Lemma 5.5, Lemma 6.5].

$\mathbf{m}=4$ : For these cases $\sqrt{J_{1}(\gamma, z)}$ does not exists as a globally defined map in the variable $\gamma \in \Gamma_{1}(-W)$, as can be seen from the occurring $\mathfrak{g}_{1}$ for the corresponding $\left(\mathfrak{g}, \mathfrak{h}, \mathfrak{g}_{1}\right)$-triples:

\begin{tabular}{|l|l|}
\hline$(\mathfrak{u}(p, q), \mathfrak{s} o(p, q), \mathfrak{s} p(n, \mathbb{R}))$ & $n$ even \\
$(\mathfrak{s} o(2, n-1) \oplus \mathfrak{s} o(2), \mathfrak{s} o(1, n-1), \mathfrak{s} o(2, n+1))$ & $n$ even \\
$(\mathfrak{s} p(n, \mathbb{R}) \oplus \mathfrak{s} p(n, \mathbb{R}), \mathfrak{s} p(n, \mathbb{R}), \mathfrak{s} p(2 n, \mathbb{R}))$ & \\
$(\mathfrak{s} o(2, q) \oplus \mathfrak{s} o(p+1), \mathfrak{s} o(1, q) \oplus \mathfrak{o}(p), \mathfrak{s} o(2, n+1))$ & $n$ even \\
\hline
\end{tabular}

From the equation

$$
\psi_{4}(\gamma \cdot(-E))=J_{1}^{2}(\gamma,-E) \psi_{4}(-E)=\left(J_{1}(\gamma,-E)\right)^{2},
$$

valid for $\gamma \in \Gamma(-W)$, it follows that we can define a holomorphic square root of $\psi_{4}$ on $\Xi(C)$. But to define the needed function $\sqrt[4]{\psi_{4}}$ we have to go to a double covering. By the above, for $(\widetilde{\gamma} \cdot(-E), x) \in \widetilde{\Xi}(C)$, i.e., $x^{4}=$ $\psi_{4}(\widetilde{\gamma} \cdot(-E))$, we have $x^{2}= \pm J_{1}(\widetilde{\gamma},-E)$. Therefore we define in this case

$$
\widetilde{\Xi}(C)_{+}:=\left\{(\widetilde{\gamma} \cdot(-E), x) \mid \widetilde{\gamma} \in \Gamma(-W), x^{2}=J_{1}(\widetilde{\gamma},-E)\right\} \subset \widetilde{\Xi}(C)
$$

and

$$
\widetilde{\Xi}(C)_{-}:=\left\{(\widetilde{\gamma} \cdot(-E), x) \mid \widetilde{\gamma} \in \Gamma(-W), x^{2}=-J_{1}(\widetilde{\gamma},-E)\right\} \subset \widetilde{\Xi}(C) .
$$

Then $\widetilde{\Gamma}(-W)$ acts on $\widetilde{\Xi}(C)_{ \pm}$as subsets of $\widetilde{\Xi}(C) \subset \widetilde{\mathcal{D}}$ by

$$
\widetilde{\gamma} \cdot(z, x)=\left(\widetilde{\gamma} \cdot z, \sqrt{J_{1}(\widetilde{\gamma}, z)} x\right) .
$$

Note that for $z \in \Xi(C)$ there are two elements $\widetilde{\gamma}, \widetilde{\delta} \in \widetilde{\Gamma}(-W)$ with $z=$ $\widetilde{\gamma} \cdot(-E)=\widetilde{\delta} \cdot(-E)$ and $\sqrt{J_{1}(\widetilde{\gamma},-E)}=-\sqrt{J_{1}(\widetilde{\delta},-E)}$. Using this, the proof of Lemma 7.3 now generalizes word-by-word to give:

\section{Lemma 7.5.}

(1) $\widetilde{\Xi}(C)_{+}=\widetilde{\Gamma}(-W) \cdot(-E, 1)$ and $\widetilde{\Xi}(C)_{-}=\widetilde{\Gamma}(-W) \cdot(-E,-1)$.

(2) We have

$$
\widetilde{\Xi}(C)=\widetilde{\Xi}(C)_{+} \dot{\cup} \widetilde{\Xi}(C)_{-}
$$

and the canonical projection $\kappa_{4}: \widetilde{\Xi}(C)_{+} \rightarrow \Xi(C)$ is a double covering, holomorphic on $\widetilde{\Xi}(C)_{+}^{o}$. 
We have on $\widetilde{G / H_{+}}:=\kappa_{4}^{-1}(G / H)$ the space of $L^{2}$-functions $L^{2}(\widetilde{G / H}+)$ and on $\widetilde{\Xi}(C)_{+}^{o}=\kappa_{4}^{-1}\left(\Xi\left(C^{o}\right)\right)$ the Hardy space $\widetilde{H}_{2}(C)_{+}$, as a space of holomorphic functions defined in the same way as $\widetilde{H}_{2}(C)$. Using roots of unity we can define the subspaces $L_{\text {odd }}^{2}\left(\widetilde{G / H_{+}}\right)$respectively $\widetilde{H}_{2 \text {,odd }}(C)_{+}$of odd functions.

Proposition 7.6. (1) The restriction from $\widetilde{\Xi}\left(C^{o}\right)$ to $\widetilde{\Xi}(C)_{+}^{o}$ induces an isomorphism of $\widetilde{\Gamma}(W)$-modules

$$
\widetilde{H}_{2, \text { odd }}(C) \simeq \widetilde{H}_{2, \text { odd }}(C)_{+} \cdot
$$

(2) The restriction from $\widetilde{G / H}$ to $\widetilde{G / H}+$ induces an isomorphism of $G$ modules

$$
L_{\text {odd }}^{2}(\widetilde{G / H}) \simeq L_{\text {odd }}^{2}(\widetilde{G / H}+) \text {. }
$$

The proof is obvious. We can in particular draw the conclusion from those considerations that we will need maximally a double covering of $\Xi(C)$ for identifying the Hardy space.

For the characterization of the image we give first the extension of $\Phi_{\delta}$ to a function on $\Phi\left(\Xi\left(C^{o}\right)\right) \subset D_{1}=G \cdot\left\{\sum x_{j^{\prime}} E_{j^{\prime}}^{\prime}\right\}$.

Lemma 7.7. For $g \in G$, define $g=k(g) \exp p(g)$ by the Cartan decomposition. Let $\left\{\nu_{\mu^{\prime}, r}\right\}$ be a base of weight vectors of $V_{\delta}$, with $\nu_{\mu^{\prime}, r}$ of weight $\mu^{\prime}$, and define $a_{\mu^{\prime}, r}$ by $\delta(k) \nu=\sum a_{\mu^{\prime}, r}\left(k^{-1}\right) \nu_{\mu^{\prime}, r}$. Identifying $\Xi\left(C^{o}\right)$ with $\Phi\left(\Xi\left(C^{o}\right)\right)$, there is a constant $c \neq 0$ such that

$$
\begin{aligned}
& \Phi_{\delta}\left(g \cdot \sum x_{j^{\prime}} E_{j^{\prime}}^{\prime}\right) \\
& =c \sum_{\mu^{\prime}, r} a_{\mu^{\prime}, r}(k(g)) \prod_{j^{\prime}} x_{j^{\prime}}^{-\mu^{\prime}\left(H_{j^{\prime}}^{\prime} / 2\right)}\left\langle\delta\left(f\left(g, \sum x_{j^{\prime}} E_{j^{\prime}}^{\prime}\right) \nu_{\mu^{\prime}, r}, \nu^{0}\right)\right\rangle,
\end{aligned}
$$

with

$$
f\left(g, \sum x_{j^{\prime}} E_{j^{\prime}}^{\prime}\right)=k_{H}\left(\operatorname{Ad}\left(\exp \left(-\frac{1}{2} \sum\left(\log x_{j^{\prime}}+\pi i\right) H_{j^{\prime}}^{\prime}\right) p(g)^{-1}\right)\right)
$$

and $\log$ the principal branch continued continously to $-\mathbb{R}^{+}$from the upper half plane.

Proof. By Equation (**) and Proposition 2.4(1) we have

$$
g \cdot \sum x_{j^{\prime}} E_{j^{\prime}}^{\prime}=\zeta_{1}\left(g \exp \left(\frac{1}{2} \sum\left(\log x_{j^{\prime}}+\pi i\right) H_{j^{\prime}}^{\prime}\right) c\right) .
$$


Therefore

$$
\begin{aligned}
& \Phi_{\delta}\left(g \cdot \sum x_{j^{\prime}} E_{j^{\prime}}^{\prime}\right) \\
& =\left\langle\nu, \check{\delta}\left(k_{H}\left(\exp \left(-\frac{1}{2} \sum\left(\log x_{j^{\prime}}+\pi i\right) H_{j^{\prime}}^{\prime}\right) g^{-1}\right)^{-1}\right) \nu^{0}\right\rangle \\
& =\left\langle\delta\left(k(g)^{-1}\right) \nu, \check{\delta}\left(\exp \left(\frac{1}{2} \sum\left(\log x_{j^{\prime}}+\pi i\right) H_{j^{\prime}}^{\prime}\right) f\left(g, \sum x_{j^{\prime}} E_{j^{\prime}}^{\prime}\right)^{-1}\right) \nu^{0}\right\rangle \\
& =c \sum_{\mu^{\prime}, r} a_{\mu^{\prime}, r}(k(g)) \prod_{j^{\prime}} x_{j^{\prime}}^{-\mu^{\prime}\left(H_{j^{\prime}}^{\prime} / 2\right)}\left\langle\delta\left(f\left(g, \sum x_{j^{\prime}} E_{j^{\prime}}^{\prime}\right)\right) \nu_{\mu^{\prime}, r}, \nu^{0}\right\rangle .
\end{aligned}
$$

For $f \in H_{2}(C)$, the inverse map to $I$ is given by extending the holomorphic function $f / \psi_{1}$ to $D_{1}$. This extension is possible if and only if $f / \psi_{1}$ is locally bounded near $D_{1} \backslash \Xi\left(C^{o}\right)$. By Theorem 3.5(5) it is enough to check this for the generating functions $f=\Phi_{\delta}$. Now by Theorem 4.3, Proposition 6.1, and the last lemma

$$
\left(\Phi_{\delta} / \Psi_{1}\right)\left(g \cdot \sum x_{j^{\prime}} E_{j^{\prime}}^{\prime}\right)=\sum_{\mu^{\prime}} b_{\mu^{\prime}}\left(g, \sum x_{j^{\prime}} E_{j^{\prime}}^{\prime}\right) \prod_{j^{\prime}} x_{j^{\prime}}^{-\left(\mu^{\prime}+\rho_{1, n}\right)\left(H_{j^{\prime}}^{\prime} / 2\right)},
$$

where $b_{\mu^{\prime}}$ is locally bounded on $G \times D_{1}$.

Theorem 7.8. $H_{\delta} \subset I\left(H_{c l}\right) \Longleftrightarrow\left(\mu+\rho_{1, n}\right)\left(H_{j^{\prime}}^{\prime}\right) \leq 0$ for all $j^{\prime}$.

Proof. Equating $\Phi_{\delta}\left(\sum x_{j^{\prime}} E_{j^{\prime}}^{\prime}\right)$ by Lemma 7.7, we get the necessary condition $\left(\mu^{\prime}+\rho_{1, n}\right)\left(H_{j^{\prime}}^{\prime}\right) \leq 0$ for all $\mu^{\prime}$ with $a_{\mu^{\prime}, r}(1) \neq 0$ and $\left\langle\nu_{\mu^{\prime}, r}, \nu^{0}\right\rangle \neq 0$. By $[\mathbf{H 8 4}$, p. 535ff] for the highest weight $\mu$ of $\delta$ both $\nu_{\mu, 1} \neq 0$ and $\left\langle\nu_{\mu, 1}, \nu^{0}\right\rangle \neq 0$, showing that the given condition is necessary.

For sufficiency, let $\mu^{\prime}$ be a weight of $\left(\delta, V_{\delta}\right)$ then $\mu^{\prime} \in \operatorname{conv}(W(\mathfrak{k}, \mathfrak{t}) \cdot \mu) \subset i \mathfrak{t}^{*}$. Applying [B88, Lemma 1.1] to the Lie algebra $\mathfrak{k}_{1 \mathbb{C}}$ with involution $-\sigma$, we can assume $W(\mathfrak{k}, \mathfrak{t}) \subset W\left(\mathfrak{k}_{1}, \mathfrak{t}_{1}\right)$, where $W\left(\mathfrak{k}_{1}, \mathfrak{t}_{1}\right)$ is the Weyl group of the root system $\Delta\left(\mathfrak{k}_{1}, \mathfrak{t}_{1}\right)$. As we already have seen $\rho_{1, n} \in\left(\mathfrak{z}\left(\mathfrak{k}_{1}\right)_{\mathbb{C}}\right)^{*} \subset\left(\mathfrak{t}_{1 \mathbb{C}}\right)^{*}$ such that now $W(\mathfrak{t}, \mathfrak{k}) \rho_{1, n}=\rho_{1, n}$ and further

$$
\left(w \cdot \mu+\rho_{1, n}\right)\left(H_{i^{\prime}}^{\prime}\right)=w \cdot\left(\mu+\rho_{1, n}\right)\left(H_{i^{\prime}}^{\prime}\right)=\left(\mu+\rho_{1, n}\right)\left(w^{-1} H_{i^{\prime}}^{\prime}\right) .
$$

We note that $i\left(\mathfrak{a} \cap C^{o}\right)=\sum \mathbb{R}^{+} H_{j^{\prime}}^{\prime} \subset i \mathfrak{a}$ is the Weyl chamber for the roots $\Delta_{1, n}^{+} \mid \mathfrak{a}$. Indeed, since $\gamma_{i}\left(H_{j}\right)=2 \delta_{i, j}$, we deduce from the Remark following Proposition 2.4 that the co-roots for $\Delta_{1, n}^{+} \mid \mathfrak{a}$ are the vectors $\left\{\frac{1}{4}\left(H_{i^{\prime}}^{\prime}+H_{j^{\prime}}^{\prime}\right) \mid 1 \leq\right.$ $\left.i^{\prime} \leq j^{\prime} \leq r^{(\prime)}\right\}$. This means that $i\left(\mathfrak{a} \cap C^{o}\right)$ is the set of all linear combinations of co-roots with positive coefficients, which - together with $\langle\alpha, \beta\rangle \geq 0$ for all $\alpha, \beta \in \Delta_{1, n}^{+} \mid \mathfrak{a}$ - implies the assertion. 
Now, as $W\left(\mathfrak{k}_{1}, \mathfrak{t}_{1}\right)$ permutes the noncompact roots, we get

$$
\left(W(\mathfrak{k}, \mathfrak{t})\left(\Delta_{1, n}^{+} \mid \mathfrak{a}\right)\right)\left|\mathfrak{a} \subset \Delta_{1, n}^{+}\right| \mathfrak{a} .
$$

Expressing this with co-roots we get $w^{-1} H_{i^{\prime}}^{\prime} \in \sum \mathbb{R}_{0}^{+} H_{j^{\prime}}^{\prime}$, such that by our equation above the given condition is sufficient for the boundedness of $\Phi_{\delta} / \Psi_{1}$.

Of course, the same condition determines the image of $\widetilde{I}$ in $\widetilde{H}_{2, \text { odd }}(C)$.

This theorem allows us now to determine the image of the classical Hardy space in $H_{2}(C)$ respectively $\widetilde{H}_{2 \text {,odd }}(C)$ as described by the root inequalities of Theorem 3.3 and Proposition 3.4.

Example. We consider $\left(\mathfrak{g}, \mathfrak{h}, \mathfrak{g}_{1}\right)=(\mathfrak{s} p(n, \mathbb{R}) \oplus \mathfrak{s} p(n, \mathbb{R}), \mathfrak{s} p(n, \mathbb{R}), \mathfrak{s} p(2 n, \mathbb{R}))$ as treated in [B97, Section 7.4]. Here

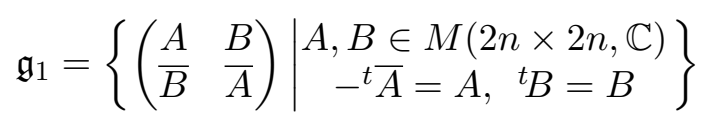

and

$$
\mathfrak{g}=\left\{\left(\begin{array}{cccc}
A_{1} & 0 & B_{1} & 0 \\
0 & A_{4} & 0 & B_{4} \\
\bar{B}_{1} & 0 & \bar{A}_{1} & 0 \\
0 & \bar{B}_{4} & 0 & \bar{A}_{4}
\end{array}\right) \in \mathfrak{g}_{1} \mid A_{i}, B_{i} \in M(n \times n, \mathbb{C})\right\} .
$$

Further, $\mathfrak{t}_{1 \mathbb{C}}^{-}$is spanned by

$$
\begin{gathered}
H_{j}=\frac{1}{2}\left(\begin{array}{cccc}
E_{j j} & E_{j j} & 0 & 0 \\
E_{j j} & E_{j j} & 0 & 0 \\
0 & 0 & -E_{j j} & -E_{j j} \\
0 & 0 & -E_{j j} & -E_{j j}
\end{array}\right), \\
H_{j+n}=\frac{1}{2}\left(\begin{array}{cccc}
E_{j j} & -E_{j j} & 0 & 0 \\
-E_{j j} & E_{j j} & 0 & 0 \\
0 & 0 & -E_{j j} & E_{j j} \\
0 & 0 & E_{j j} & -E_{j j}
\end{array}\right)
\end{gathered}
$$

for $j=1, \ldots, n$, whereas a base for $\mathfrak{a}_{\mathbb{C}}$ is given by by the vectors $H_{j}^{\prime}=$ $H_{j}+H_{j+n}$. Since $\mathfrak{g}_{1}$ is a normal Lie algebra the multiplicity of all roots in $\Delta_{1}$ is one. Defining $\gamma_{j}^{\prime}:=\frac{1}{2}\left(\gamma_{j}+\gamma_{j+n}\right)$ we get by restricting the roots of $\Delta_{1}^{+}$ to $\mathfrak{a}_{\mathbb{C}}$

$$
\Delta^{+}=\left\{\frac{1}{2}\left(\gamma_{i}^{\prime}+\gamma_{j}^{\prime}\right) \mid 1 \leq i \leq j \leq n\right\} \cup\left\{\frac{1}{2}\left(\gamma_{i}^{\prime}-\gamma_{j}^{\prime}\right) \mid 1 \leq i<j \leq n\right\},
$$

and all these roots have multiplicity 2. With $\Delta_{n}^{+}=\left\{\frac{1}{2}\left(\gamma_{i}^{\prime}+\gamma_{j}^{\prime}\right) \mid 1 \leq i \leq\right.$ $j \leq n\}$ by Proposition 2.4(6) and the definition of $c_{\text {min }}$ we get $\mathfrak{a} \cap C=c_{\min }$. Therefore, by the Remark following Proposition 3.4 the Hardy space $\widetilde{H}_{2}(C)$ comprises the whole holomorphic discrete series. To describe these in more 
detail, with $\mu$ the highest weight of $\delta$ with respect to $\Delta_{k}^{+}=\left\{\frac{1}{2}\left(\gamma_{i}^{\prime}-\gamma_{j}^{\prime}\right) \mid 1 \leq\right.$ $i<j \leq n\}$ we have for $H_{\delta} \subset \widetilde{H}_{2, \text { odd }}(C)$

$$
\mu=\sum m_{j} \gamma_{j}^{\prime}, \quad m_{1} \geq m_{2} \geq \ldots \geq m_{n}, \quad m_{j} \in \mathbb{Z}+\frac{1}{2} .
$$

To apply Theorem 3.3(1) we calculate

$$
\begin{aligned}
\rho & =\frac{1}{2} \sum_{\alpha \in \Delta^{+}} \operatorname{dim} \mathfrak{g}_{\mathbb{C}, \alpha} \alpha=\sum_{1 \leq i \leq j \leq n} \frac{1}{2}\left(\gamma_{i}^{\prime}+\gamma_{j}^{\prime}\right)+\sum_{1 \leq i<j \leq n} \frac{1}{2}\left(\gamma_{i}^{\prime}-\gamma_{j}^{\prime}\right) \\
& =(n+1) \sum_{j=1}^{n} \gamma_{j}^{\prime}-\sum_{j=1}^{n} j \gamma_{j}^{\prime} .
\end{aligned}
$$

Inserting into $\langle\mu+\rho, \alpha\rangle<0$, for $\alpha \in \Delta_{n}^{+}$, we derive the condition

$$
H_{\delta} \subset \widetilde{H}_{2, \text { odd }}(C) \Longleftrightarrow-n>m_{1} \geq m_{2} \geq \ldots \geq m_{n} .
$$

We show next that $\widetilde{I}$ is actually surjective. In fact, with

$$
\rho_{1, n}=\frac{1}{2}\left(\frac{2 n+1}{2}\right)\left(\gamma_{1}+\cdots+\gamma_{2 n}\right)
$$

the condition of Theorem 7.8 reduces to

$$
\left\langle\mu, \gamma_{j}^{\prime}\right\rangle \leq-\frac{2 n+1}{4} \quad \text { or } \quad m_{j} \leq-\frac{2 n+1}{2}
$$

such that, $m_{j}+\frac{1}{2}$ being an integer, the embedding $\widetilde{I}$ is onto. Another discussion of this example can be found in [KØ97].

Similar to the given example this "best possible" case, with $H_{2}(C)$ respectively $\widetilde{H}_{2 \text {,odd }}(C)$ being the direct sum of all holomorphic discrete series representations and $I$ respectively $\widetilde{I}$ onto, occurs for the $\left(\mathfrak{g}, \mathfrak{h}, \mathfrak{g}_{1}\right)$-triples

$$
\begin{aligned}
& (\mathfrak{s} p(n, \mathbb{R}) \oplus \mathfrak{s} p(n, \mathbb{R}), \mathfrak{s} p(n, \mathbb{R}), \mathfrak{s} p(2 n, \mathbb{R})) \\
& (\mathfrak{s} p(2 n, \mathbb{R}), \mathfrak{s} p(n, \mathbb{C}), \mathfrak{s} u(2 n, 2 n)) \\
& (\mathfrak{g}, \mathfrak{h}, \mathfrak{g} \times \mathfrak{g}) \text { for }(\mathfrak{g}, \mathfrak{h}) \text { of Cayley type } \\
& (\mathfrak{s} o(2, n), \mathfrak{s} o(1, n), \mathfrak{s} o(2, n+1)) .
\end{aligned}
$$

Note that these are exactly the cases with $\mathfrak{a} \neq \mathfrak{t}_{1}^{-}$.

Example. We discuss next the compactifications described by [B97, Cor. 5.3]. These are the $\left(\mathfrak{g}, \mathfrak{h}, \mathfrak{g}_{1}\right)$-triples

$$
\begin{aligned}
& (\mathfrak{s}(\mathfrak{u}(p, q) \oplus \mathfrak{u}(p, q)), \mathfrak{s} u(p, q), \mathfrak{s} u(n, n)),\left(\mathfrak{u}(2 p, 2 q), \mathfrak{s} p(p, q), \mathfrak{s} o^{*}(4 n)\right), \\
& (\mathfrak{u}(p, q), \mathfrak{s} o(p, q), \mathfrak{s} p(n, \mathbb{R})),(\mathfrak{s} o(2, n-1) \oplus \mathfrak{s} o(2), \mathfrak{s} o(1, n-1), \mathfrak{s} o(2, n+1)), \\
& \left(\mathfrak{e}_{6(-14)} \oplus \mathfrak{s} o(2), \mathfrak{f}_{4(-20)}, \mathfrak{e}_{7(-25)}\right) .
\end{aligned}
$$


Here $\gamma_{j^{\prime}}^{\prime}=\gamma_{j^{\prime}}$, i.e., $H_{j^{\prime}}^{\prime}=H_{j^{\prime}}$, and $\mathfrak{z}(\mathfrak{g})=\sum \epsilon_{j} i H_{j}$, where without loss of generality $\epsilon_{j}=1$ for $j \leq p$ and $\epsilon_{j}=-1$ for $j>p$. By the explicit form of

$$
\sigma=\operatorname{Ad}\left(\exp \frac{\pi}{2} \sum \epsilon_{j} i H_{j}\right)
$$

the action of this involution on the root spaces $\mathfrak{g}_{1 \mathbb{C}, \alpha}$ can be determined easily and gives us

$$
\begin{aligned}
\Delta_{k}=\left\{ \pm \frac{1}{2}\left(\gamma_{i}-\gamma_{j}\right) \mid i<j \leq p \text { or } p<i<j\right\} \text { and } & \\
\Delta_{n} & =\left\{ \pm \frac{1}{2}\left(\gamma_{i}+\gamma_{j}\right) \mid i \leq p<j\right\} .
\end{aligned}
$$

The weight spaces for $\gamma \in \Delta$ are the same as for $\gamma \in \Delta_{1}$, especially all have the same dimension $d$, such that

$$
\rho=\frac{d}{4}\left[\sum_{i<j \leq p}\left(\gamma_{i}-\gamma_{j}\right)+\sum_{p<i<j}\left(\gamma_{i}-\gamma_{j}\right)+\sum_{i \leq p<j}\left(\gamma_{i}+\gamma_{j}\right)\right]=\frac{d}{4}(r+1) \sum_{j=1}^{r} \gamma_{j} .
$$

With $\Delta_{k}^{+}=\left\{\frac{1}{2}\left(\gamma_{i}-\gamma_{j}\right)\right\}$ the highest weight has the form $\mu=\sum n_{j} \gamma_{j}$ with $n_{1} \geq n_{2} \geq \ldots \geq n_{p}$ and $n_{p+1} \geq \ldots \geq n_{r}$. Alternatively, with $\mu=$ $d \chi_{\delta}+\mu_{s}$ we can describe the highest weight with $d \chi_{\delta}=\lambda\left(\frac{1}{2 r} \sum \epsilon_{j} \gamma_{j}\right)$ and $\mu_{s}=\sum s_{j} \gamma_{j}$, where $\lambda \in \mathbb{R}$ and the $s_{j}$ are integers with $s_{1} \geq s_{2} \geq \ldots \geq s_{p}$ and $s_{p+1} \geq \ldots \geq s_{r}$. (The $s_{j}$ are also restricted by $\sum \epsilon_{j} s_{j}=0$, since $\mu_{s}$ is zero on $\mathfrak{z}(\mathfrak{g})$.) As $d \chi_{\delta}$ integrates to a representation $\chi_{\delta} \in \hat{Z}$ respectively $\tilde{\chi}_{\delta} \in \hat{\widetilde{Z}}$ we have $\lambda \in \mathbb{Z}$ respectively $\lambda \in \frac{1}{2} \mathbb{Z}$. Finally, to get a representation $\delta=\chi_{\delta} \otimes \delta_{s}$ the representations $\chi_{\delta}$ and $\delta_{s}$ must be equal on the central subgroup $D=Z \cap G_{s}$ respectively $\widetilde{D}$ for the covering group. We call this the $(D)$-condition.

With $\rho$ as above we get from Theorem 3.3(1)

$$
n_{i}+n_{j}<-\frac{d}{2}(r+1), \quad \text { for } i \leq p<j,
$$

which is by the form of $\mu$ equivalent to $n_{1}+n_{p+1}<-\frac{1}{2}(r+1)$, and from Proposition 3.4 the condition of $W$-admissibility

$$
n_{j} \leq 0, \quad \text { for } j=1, \ldots, r .
$$

To summarize, the Hardy space $H_{2}(C)\left(\widetilde{H}_{2}(C)\right)$ is determined by $\left(n_{1}, \ldots\right.$, $\left.n_{r}\right) \in \mathbb{Z}^{r}+\left(\frac{1}{2} \mathbb{Z}(1, \ldots, 1)\right)$ with

(1) the $(D)$-condition,

(2) $0 \geq n_{1} \geq \ldots \geq n_{p}$ and $0 \geq n_{p+1} \geq \ldots \geq n_{r}$,

(3) $n_{1}+n_{p+1}<-\frac{d}{2}(r+1)$. 
For the covering case $\widetilde{H}_{2 \text {,odd }}(C)$ is the subspace spanned by those holomorphic discrete series representations which are not representations for $G$.

We remark that this Hardy space is strictly smaller than the Hardy space $H_{2}\left(C_{\text {min }}\right)$ respectively $\widetilde{H}_{2 \text {,odd }}\left(C_{\text {min }}\right)$. Indeed, by Theorem 3.3 , Proposition 3.4, and the following Remark, it is clear that this "maximal" Hardy space is the direct sum $\bigoplus_{\delta_{s}, \chi \in \hat{Z}\left(\delta_{s}\right)} \mathbb{C}_{\chi} \otimes H_{\delta_{s}}$, where the $\delta_{s}$ exhaust the holomorphic discrete series and $\hat{Z}\left(\delta_{s}\right)$ is the set of unitary characters which fulfill with $\delta_{s}$ the $(D)$-condition, i.e., $\chi \otimes \delta_{s}$ is a representation of $G$ respectively $\widetilde{G}$. To see that this is a bigger space than $H_{2}(C)$ we calculate, with $\Delta_{n}^{+}$as above,

$$
\mathfrak{a} \cap C_{\min }^{o}=-i \sum_{a \leq p<b} \mathbb{R}^{+}\left(H_{a}+H_{b}\right) .
$$

Therefore the condition of admissibility for the minimal cone is $n_{a}+n_{b} \leq 0$, for $a \leq p<b$, or equivalently $n_{1}+n_{p+1} \leq 0$ compared with $n_{1} \leq 0$ and $n_{p+1} \leq 0$ for the cone $C$.

The image of the classical Hardy space is described by

$$
n_{j} \leq-\frac{1}{2}\left(1+\frac{d(r-1)}{2}\right), \text { for } j=1, \ldots, r .
$$

To give a more explicit example, we specialize further to the case $G / H=$ $S(U(1,1) \times U(1,1)) / S U(1,1)$, cf. [B97, Section 6.5] for the realization. Here $H_{j}=\left(E_{j, j}+E_{j+2, j+2}\right) \in \mathfrak{s} u(2,2)_{\mathbb{C}}=\mathfrak{s l}(4, \mathbb{C})$ and

$$
\mathfrak{a}=\mathfrak{t} \cap \mathfrak{q}=\mathbb{R}\left(\begin{array}{cccc}
i & & & \\
& -i & & \\
& & -i & \\
& & & i
\end{array}\right)+\mathbb{R}\left(\begin{array}{llll}
i & & & \\
& i & & \\
& & -i & \\
& & & -i
\end{array}\right),
$$

where the first summand is equal to $\mathfrak{z}(\mathfrak{g})$. Let $\mu \in i(\mathfrak{t} \cap \mathfrak{q})^{*}$ be the highest weight for a holomorphic discrete series representation of $G / H$. Identifying $\mathfrak{t} \cap \mathfrak{q}$ with its dual, we write

$$
\mu=a\left(\begin{array}{cccc}
1 & & & \\
& -1 & & \\
& & -1 & \\
& & & 1
\end{array}\right)+b\left(\begin{array}{cccc}
1 & & & \\
& 1 & & \\
& & -1 & \\
& & & -1
\end{array}\right)
$$

such that the first summand gives the central character $d \chi_{\delta}$ and the second $\mu_{s}$. We have the integrability conditions $a, b \in \mathbb{Z}$, and the $(D)$-condition gives $a+b \in 2 \mathbb{Z}$. Further

$$
\Delta^{+}=\Delta_{n}^{+}=\left\{\frac{1}{2}\left(\gamma_{1}+\gamma_{2}\right)\right\}
$$

and $\rho=\frac{1}{2}\left(\gamma_{1}+\gamma_{2}\right)$. Thus Theorem 3.3(1) gives us $b<-1$ and of course no restriction on the "central parameter" $a$. 
However we have also the condition of $W$-admissibility. Rewritting this in terms of the parameters $a$ and $b$ shows that $W$-admissibility is equivalent to $|a| \leq-b$.

For the determination of the image of the classical Hardy space in $H_{2}(C)$ it is straightforward from the definition that $\rho_{1, n}=H_{1}+H_{2}$ (with our identification of $\mathfrak{t}$ and $\left.\mathfrak{t}^{*}\right)$. Then the determining equations $\left(\mu+\rho_{1, n}\right)\left(H_{i}\right) \leq 0$ are equivalent to $|a| \leq-b-1$. The image of the classical Hardy space therefore misses the two half lines $a+b=0$ and $a-b=0$. We remark that this differs from [KØ96, Figure 3] where the missing half lines lie on one side of the cone.

One can deal with the remaining cases as above. With notation as in the preceding examples, we summarize the results as follows:

$\left(\mathfrak{g}, \mathfrak{h}, \mathfrak{g}_{1}\right)=\left(\mathfrak{s} o^{*}(2 n) \oplus \mathfrak{s} o^{*}(2 n), \mathfrak{s} o^{*}(2 n), \quad \mathfrak{s} o^{*}(4 n)\right)$ : The Hardy space $H_{2}(C)=H_{2}\left(C_{\min }\right)$ is parametrized by $0 \geq n_{1} \geq \ldots \geq n_{n}$ with $n_{i}+n_{j}<$ $-2 n+i+j$, for $i \neq j$, and $I\left(H_{c l}\right)$ is given by $n_{1} \leq-n$.

$\left(\mathfrak{g}, \mathfrak{h}, \mathfrak{g}_{1}\right)=\left(\mathfrak{s} o^{*}(2 n), \mathfrak{s} o(n, \mathbb{C}), \mathfrak{s} u(n, n)\right)$ : The Hardy space $H_{2}(C)=$ $H_{2}\left(C_{\text {min }}\right)$ is parametrized by $0 \geq n_{1} \geq \ldots \geq n_{n}$ with $n_{i}+n_{j}<-n+\frac{i+j}{2}$, for $i \neq j$, and $I\left(H_{c l}\right)$ is given by $n_{1} \leq-\frac{n}{2}$.

$\left(\mathfrak{g}, \mathfrak{h}, \mathfrak{g}_{1}\right)=(\mathfrak{s} o(2, q) \oplus \mathfrak{s} o(p+1), \mathfrak{s} o(1, q) \oplus \mathfrak{o}(p), \mathfrak{s} o(2, n+1)): H_{2}(C)$ respectively $\widetilde{H}_{2, \text { odd }}(C)$ is parametrized by $0 \geq n_{1} \geq n_{2}$ with $n_{1}+n_{2} \leq$ $-\frac{1}{4}(n+q-p)$ and $I\left(H_{c l}\right)$ or $\widetilde{I}\left(H_{c l}\right)$ is given by $n_{1} \leq-\frac{1}{4}(n+1)$. Note that for this case the decomposition of $(\mathfrak{g}, \mathfrak{h}, \tau)$ into irreducible symmetric algebras gives not a decomposition of the causal structure.

We remark at the end that our results give now the Cauchy-Szegö kernel $K_{I}(\cdot, \cdot)$ for the subspace $I\left(H_{c l}\right) \subset H_{2}(C)$ respectively $\widetilde{I}\left(H_{c l}\right) \subset \widetilde{H}_{2 \text {,odd }}(C)$. Indeed, with $\left\{\varphi_{n}\right\}$ an orthonormal base of $H_{2}(C)$ the kernel is generally given by

$$
K(z, w)=\sum \varphi_{n}(z) \overline{\varphi_{n}(w)}
$$

such that

$$
K_{I}(z, w)=\sqrt[m]{\psi_{m}(z)} K_{c l}(z, w) \sqrt{\sqrt[m]{\psi_{m}(w)}}
$$

where the kernel $K_{c l}$ for the classical Hardy space is known ([FK94, Prop. $\mathrm{X} .1 .3])$. Note also that this kernel is invariant by deck transformations of the covering $\widetilde{\Xi}(C) \rightarrow \Xi(C)$. Therefore, when we have an isomorphism of Hardy spaces as in Proposition 7.4, we get the corresponding image of the kernel by simply projecting down.

\section{References}

[B88] E.P. van den Ban, The principal series for a reductive symmetric space I. Hfixed distribution vectors, Ann. Scient. Éc. Norm. Sup., 21 (1988), 359-412, MR 90a:22016, Zbl 714.22009. 
[Bal] W. Bertram and J. Hilgert, Hardy spaces and analytic continuation of Bergman spaces, Bull. Soc. Math. France, 126 (1998), 435-482, MR 2000a:32012, Zbl 920.22006.

[B97] F. Betten, Causal compactification of compactly causal spaces, Trans. A.M.S., to appear.

[BD92] J.-L. Brylinski and P. Delorme, Vecteurs distributions H-invariants pour les séries principales généralisées d'espaces symetriques reductifs et prolongement meromorphe d'intégrales d'Eisenstein, Invent. Math., 109 (1992), 619-664, MR 93m:22016, Zbl 785.22014.

[C96] M. Chadli, Espace de Hardy d'un Espace Symétrique de Type Cayley, Thesis, Paris, 1996, MR 97f:43013, Zbl 860.46036.

[FK94] J. Faraut and A. Korányi, Analysis on Symmetric Cones, Clarendon Press, Oxford, 1994, MR 98g:17031, Zbl 841.43002.

[H78] S. Helgason, Differential Geometry, Lie Groups, and Symmetric Spaces, Academic Press, New York, 1978, MR 80k:53081, Zbl 451.53038.

[H84] _ Groups and Geometric Analysis. Integral Geometry, Invariant Differential Operators and Spherical Functions, Academic Press, New York, 1984, MR 86c:22017, Zbl 543.58001.

[H94] - Geometric Analysis on Symmetric Spaces, A.M.S., Providence, Rhode Island, 1994, MR 96h:43009, Zbl 809.53057.

[HÓ92] J. Hilgert and G. Ólafsson, Analytic extension of representations, the solvable case, Japan J. Math., 18 (1992), 213-290, MR 94f:22011.

[HÓ96] _ Causal Symmetric Spaces, The Geometry and Harmonic Analysis, Perspectives in Math., 18, Academic Press, New York, 1996, MR 97m:43006.

[HÓØ91] J. Hilgert, G. Ólafsson and B. Ørsted, Hardy spaces on affine symmetric spaces, J. Reine Angew. Math., 415 (1991), 189-218, MR 92h:22030, Zbl 716.43006.

[KW65] A. Korányi and J.A. Wolf, Realization of Hermitian symmetric spaces as generalized half planes, Ann. of Math., 81 (1965), 265-288, MR 30 \#4980, Zbl 137.27402.

[KØ96] K. Koufany and B. Ørsted, Function spaces on the Ol'shanskiu semigroup and the Gelf'and-Gindikin program, Anal. Inst. Fourier, 46 (1996), 689-722, MR 97k:22021, Zbl 853.22010.

[KØ97] _ Hardy spaces on two sheeted covering semigroups, J. Lie Theory, 7 (1997), 245-267, MR 98k:22060, Zbl 884.22006.

[KN96] B. Krötz and K.-H. Neeb, On hyperbolic cones in mixed symmetric spaces, J. of Lie Theory, 6 (1996), 69-146, MR 97k:17007, Zbl 860.22004.

[KNÓ97] B. Krötz, K.-H. Neeb and G. Ólafsson, Spherical representations and mixed symmetric spaces, Representation Theory, 1 (1997), 424-461, MR 99a:22031, Zbl 887.22022.

[L69] O. Loos, Symmetric Spaces, I: General Theory, W.A. Benjamin Inc., New York, 1969, MR 39 \#365b, Zbl 175.48601.

[M79] T. Matsuki, The orbits of affine symmetric spaces under the action of minimal parabolic subgroups, J. Math. Soc. Japan, 31 (1979), 331-357, MR 81a:53049, Zbl 396.53025. 
[M97] V.F. Molchanov, Holomorphic discrete series for hyperboloids of Hermitian type, J. Funct. Anal., 147 (1997), 26-50, MR 99b:22026, Zbl 881.43005.

[Ó84] G. Ólafsson, Die Langlands-Parameter für die Flensted-Jensensche fundamentale Reihe, Math. Scand., 55 (1984), 229-244, MR 86k:22034, Zbl 561.22008.

[Ó87]_ Fourier and Poisson transformation associated to a semisimple symmetric space, Invent. Math., 90 (1987), 605-629, MR 89d:43011, Zbl 665.43004.

[Ó91] _ Symmetric spaces of Hermitian type, Diff. Geom. and Appl., 1 (1991), 195-233, MR 94g:22034, Zbl 785.22021.

[Ó97a] _ Spherical functions and spherical Laplace transform on ordered symmetric spaces, submitted, preprint: http://www.math.lsu.edu/ olafsson.

[Ó97b] _ Open problems in harmonic analysis on causal symmetric spaces, in 'Positivity in Lie Theory: Open Problems', eds. J. Hilgert, J.D. Lawson, K.-H. Neeb and E.B. Vinberg, Expositions in Mathematics, De Gruyter, Berlin, 1998, 249-270, MR 99h:43021, Zbl 919.43006.

[Ó00] Analytic continuation in representation theory and harmonic analysis, in 'Seminaires et Congres', 4, Global Analysis and Harmonic Analysis, eds. J.P. Bourguigon, T. Branson and O. Hijazi, French Mathematical Society, 2000, 201-233.

[ÓØ88] G. Ólafsson and B. Ørsted, The holomorphic discrete series for affine symmetric spaces, I, J. Funct. Anal., 81 (1988), 126-159, MR 89m:22021, Zbl 678.22008.

[ÓØ91] _ The holomorphic discrete series of an affine symmetric space and representations with reproducing kernels, Trans. A.M.S., 326 (1991), 385-405, MR 91j:22014, Zbl 760.22020.

[ÓØ99] — Causal compactification and Hardy spaces, Trans. A.M.S., 351 (1999), 3771-3792, MR 99m:22005, Zbl 928.43007.

[O95] G.I. Ol'shanskiŭ, Cauchy-Szegö kernels for Hardy spaces on simple Lie groups, J. Lie Theory, 5 (1995), 241-273, Zbl 860.43008.

[SW71] E.M. Stein and G. Weiss, Fourier Analysis on Euclidean Spaces, Princeton University Press, Princeton, New Jersey, 1971, MR 46 \#4102, Zbl 232.42007.

Received December 29, 1998. The second author was supported by NSF grant DMS9626541, DMS-0070607, and LEQSF (1996-99)-RD-A-12.

\section{UNIVERsitätT GÖTtINGEN}

Mathematisches Institut

Bunsenstrasse 3-5, D-37073 Göttingen

Germany

E-mail address: betten@cfgauss.uni-math.gwdg.de

Department of Mathematics

LOUisiana State University

BAton Rouge, LA 70803

E-mail address: olafsson@math.lsu.edu 